\title{
V3885 Sagittarius: a Comparison with a Range of Standard Model Accretion Disks ${ }^{1}$
}

\author{
Albert P. Linnell ${ }^{2}$, Patrick Godon ${ }^{3}$, Ivan Hubeny ${ }^{4}$, Edward M. Sion ${ }^{5}$, Paula Szkody ${ }^{6}$, and \\ Paul E. Barrett ${ }^{7}$ \\ ${ }^{2}$ Department of Astronomy, University of Washington, Box 351580, Seattle, WA \\ 98195-1580 \\ ${ }^{3}$ Department of Astronomy and Astrophysics, Villanova University, Villanova, PA 19085 \\ ${ }^{4}$ Steward Observatory and Department of Astronomy, University of Arizona, Tucson, AZ \\ 85721 \\ ${ }^{5}$ Department of Astronomy and Astrophysics, Villanova University, Villanova, PA 19085 \\ ${ }^{6}$ Department of Astronomy, University of Washington, Box 351580, Seattle, WA \\ 98195-1580 \\ ${ }^{7}$ United States Naval Observatory, Washington, DC 20392

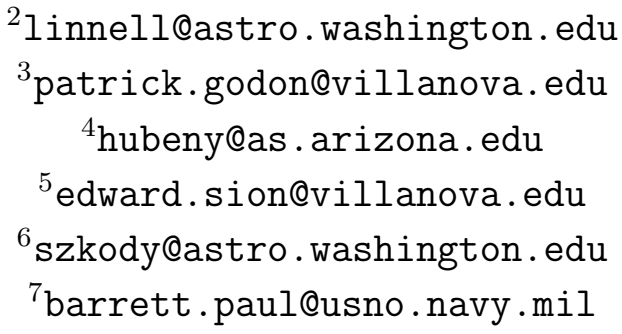

\begin{abstract}
A $\widetilde{\chi}^{2}$ analysis of standard model accretion disk synthetic spectrum fits to combined FUSE and STIS spectra of V3885 Sagittarius, on an absolute flux basis, selects a model that accurately represents the observed SED. Calculation of the synthetic spectrum requires the following system parameters. The cataclysmic variable secondary star period-mass relation calibrated by Knigge in 2007 sets the secondary component mass. A mean white dwarf (WD) mass from the same study, that is consistent with an observationally-determined mass ratio, sets the adopted WD mass of $0.7 M_{\odot}$, and the WD radius follows from standard theoretical models. The adopted inclination, $i=65^{\circ}$, is a literature consensus, and is
\end{abstract}

\footnotetext{
${ }^{1}$ Based on observations made with the NASA/ESA Hubble Space Telescope, obtained at the Space Telescope Science Institute, which is operated by the Association of Universities for Research in Astronomy, Inc. under NASA contract NAS5-26555, and the NASA-CNES-CSA Far Ultraviolet Explorer, which is operated for NASA by the Johns Hopkins University under NASA contract NAS5-32985
} 
subsequently supported by $\widetilde{\chi}^{2}$ analysis. The mass transfer rate is the remaining parameter to set the accretion disk $T_{\text {eff }}$ profile, and the Hipparcos parallax constrains that parameter to $\dot{M}=5.0 \pm 2.0 \times 10^{-9} M_{\odot} \mathrm{yr}^{-1}$ by a comparison with observed spectra. The fit to the observed spectra adopts the contribution of a $57,000 \pm 5000 \mathrm{~K}$ WD. The model thus provides realistic constraints on $\dot{M}$ and $T_{\text {eff }}$ for a large $\dot{M}$ system above the period gap.

Subject headings: Stars:Novae,Cataclysmic Variables,Stars:White Dwarfs,Stars: Individual:Constellation Name: V3885 Sagittarius

\section{Introduction}

Cataclysmic variables (CVs) are semi-detached binary stars in which a late main sequence star loses mass onto a white dwarf (WD) by Roche lobe overflow (Warner 1995). In non-magnetic systems the mass transfer stream produces an accretion disk with mass transport inward and angular momentum transport outward, driven by viscous processes. The accretion disk may extend inward to the WD; the outer boundary extends to a tidal cutoff limit imposed by the secondary star in the steady state case. If the mass transfer rate is below a certain limit, the accretion disk is unstable and undergoes brightness cycles (outbursts), and if above the limit, the accretion disk is stable against outbursts. The latter objects (which have no recorded outburst of any type) are called nova-like (NL) systems. As shown by current studies (Ribiero \& Diaz (2007); Hartlev et al. (2005, 2002); Woods et al. (1992); Metz (1989); Haug \& Dreschel (1985); Guinan \& Sion (1982); Cowley \& Crampton (1977)) V3885 Sgr is a NL system (see Warner 1995 for a detailed discussion). V3885 Sgr has a Hipparcos (Perrvman et al. 1997) parallax of $9.11 \pm 1.95$ mas. The orbital period is $0.20716071^{\mathrm{d}}$ (Ribiero \& Diaz 2007) and the inclination $i \sim 65^{\circ}$ (Haug \& Dreschel 1985; Hartley et al. 2005; Ribiero \& Diaz 2007).

NL systems are of special interest because they are expected to have an accretion disk radial temperature profile given by an analytic expression (Frank. King \& Raine 1992, eq.5.41) (hereafter FKR) which defines the so-called standard model; the expression includes the mass transfer rate $\dot{M}$ as an explicit variable. In NL systems, which are above the period gap (Howell, Nelson, \& Rappaport 2001), the accretion disk dominates the system spectrum. Fitting a synthetic spectrum, based on the analytic model, a proxy of the accretion disk temperature profile, to an observed spectrum potentially determines $\dot{M}$, a physical parameter of basic importance since it controls the evolution of CV systems (Howell. Nelson. \& Rappaport 2001). But the analytic expression also is an explicit function of the WD mass, $M_{\mathrm{wd}}$, and it must be determined independently. 
By the basic paradigm of CV evolution (Howell, Nelson, \& Rappaport 2001) there is a close relation between orbital period and donor mass. In a recent paper, Knigge (2007) has calibrated the relations among $P, M_{2}, R_{2}, T_{\text {eff, },}$, and donor spectral type. Thus, given an orbital perod below the tabular upper limit of $6^{\mathrm{h}}, M_{2}$ can be determined independently of other parameters. If there is an observationally-determined mass ratio, the WD mass follows directly.

A normalizing factor is necessary in comparing synthetic spectra with observed spectra and the factor depends on the distance to the system. If the distance is known independently an absolute determination of $\dot{M}$ potentially is possible.

A complication in fitting a synthetic spectrum to an observed spectrum is the fact that the WD may contribute detectably to the system spectrum; evaluating the WD contribution requires the WD $T_{\text {eff. }}$ A limited number of CV systems have known values for their WD $T_{\text {eff }}$ (Sion 1999; Winter \& Sion 2003) with extremely few being NLs. Because of the large $\dot{M}$ in NL systems, the accretion disk dominates the system spectrum in the UV and optical, and makes determination of the WD $T_{\text {eff }}$ difficult. Thus, the $T_{\text {eff }}$ of an additional NL WD is of obvious interest.

\section{The FUSE and STIS Spectra}

Table 1 presents the observations log. The STIS spectra are the same as three STIS spectra described by Hartley et al. (2002).

\subsection{The FUSE Spectrum}

FUSE is a low-earth orbit satellite, launched in June 1999. Its optical system consists of four optical telescopes (mirrors), each separately connected to a different Rowland spectrograph. The four diffraction gratings of the four Rowland spectrographs produce four independent spectra on two microchannel plates (detectors). Two mirrors and two gratings are coated with $\mathrm{SiC}$ to provide wavelength coverage below $1020 \AA$, while the other two mirrors and gratings are coated with $\mathrm{Al}$ and $\mathrm{LiF}$. The $\mathrm{Al}+\mathrm{LiF}$ coating provides about twice the reflectivity of $\mathrm{SiC}$ at wavelengths $>1050 \AA$, and very little reflectivity below $1020 \AA$. These are known as the $\mathrm{SiC} 1, \mathrm{SiC} 2, \mathrm{LiF} 1$ and $\mathrm{LiF} 2$ channels producing a total of 8 (partially overlapping) spectral segments: SiC1a, SiC1b, SiC2a, SiC2b, LiF1a, LiF1b, LiF2a, LiF2b for each exposure.

Four FUSE observations of V3885 Sgr prospectively are available for analysis. An 
observation on Aug. 10, 2002 has a short exposure time and has inferior S/N ratio compared to other exposures. An exposure on 24 May 2000 (UT:01:51:55) has the longest exposure time and occurs within 25 days of our chosen STIS spectrum. We chose this spectrum for analysis. The exposure used the 30"x30" LWRS Large Square Aperture in TIME TAG mode. The telescope collected 11 exposures obtained during 11 consecutive orbits of the FUSE spacecraft, totaling 12865 s of raw exposure time for the 88 spectral segments; we downloaded the spectra from the MAST archive and processed them as described below.

The data were processed with the latest and final version of CalFUSE (v3.2.1) (Dixon et al. 2007). The main change from previous versions of CalFUSE is that the data are maintained as a photon list (the intermediate data file - IDF) throughout the pipeline. Bad photons are flagged but not discarded, so the user can examine, filter, and combine data without rerunning the pipeline. Event bursts (short periods during an exposure when high count rates are registered on one or more detectors) are processed automatically in this latest version of CalFUSE. The bursts exhibit a complex pattern on the detector, their cause, however, still is unknown (it has been confirmed (Dixon et al. 2007) that they are not detector effects).

The 11 exposures all have essentially identical continuum flux levels and there is no detectable change in flux from exposure to exposure. The main features that vary from exposure to exposure are the depth, width and shape of the absorption lines. The next step is to coadd the 11 exposures, for the 8 individual segments. The count rate indicates screening of $22 \mathrm{~s}$ of high voltage $(\mathrm{HV})$ for the 8 spectral segments. In addition there was a loss of good exposure time due to event bursts of 472s, 409s, 390s, and 511s in segments 1a's, 1b's, 2a's and 2b's respectively. We obtain a total of 12310s, 12373s, 12392s and 12271s for segments 1a's, 1b's, 2a's and 2b's, respectively, including more than 8200s of NIGHT time (i.e. when the spacecraft is located in the shadow of the earth). Since the total good collection time varies, we weighted the segments accordingly (see below).

During the observations, Fine Error Sensor A (which images the LiF1 aperture) was used to guide the telescope. The spectral regions covered by the spectral channels overlap, and these overlap regions are then used to renormalize the spectra in the SiC1 (a \& b), LiF2 (a \& b), and SiC2 (a \& b) channels to the flux in the LiF1 (a \& b) channel. The low sensitivity portions (usually the edges) of each channel are discarded. In addition, there exists a narrow dark stripe of decreased flux in the spectra running in the dispersion direction, affectionally identified as the "worm", which can attenuate as much as $50 \%$ of the incident light in the affected portions of the spectrum, due to shadows of the wires in a grid above the detector. Because of the temporal changes in the strength and position of the worm, CalFUSE (Dixon et al. 2007) cannot correct target fluxes for its presence. Therefore, we carried out a visual inspection of the FUSE channels to locate the worm and we manually 
discarded the portion of the spectrum affected by the worm. : 1130-1189 $\AA$ in the LiF $1 b$ channel.

We combined the individual exposures and channels to create a time-averaged spectrum, weighting the flux in each output datum by the exposure time (as mentioned earlier) and sensitivity of the input exposure and channel of origin. The final product is a spectrum that covers almost the full FUSE wavelength range $905 \AA-1187 \AA$; because we disregarded that segment of the spectrum affected by the worm, the spectrum ends at $\sim 1182 \AA$. The absorption lines in the final spectrum are blue shifted by about $0.4 \AA$. Based on the Ribiero \& Diaz (2007) ephemeris, the FUSE spectrum extends from orbital phase 0.29 to orbital phase 1.01.

\subsection{The STIS spectra}

The STIS spectra (from the MAST archive) were taken in TIME-TAG mode, with the FUV-MAMA detector. The E140M echelle grating was used (with a central wavelength of $1425 \AA$ ), and the $0.2 \mathrm{x} 0.2$ aperture. The STIS spectra were processed with CALSTIS version 2.22. The calibrations were performed using the STSDAS package. Each STIS spectrum includes 44 echelle spectra, each one covering roughly $18 \AA$. The echelle spectra overlap except in the longer wavelengths. As a consequence when the echelle spectra are merged to produce the final spectrum, there are 4 gaps in the longer wavelengths. The gaps are about $\sim 0.5 \AA$ wide and are at intervals of about $18 \AA$. We have removed the gaps by hand.

The first and third STIS spectrum have closely similar continua and differ slightly in the shape of their absorption lines, with a blue shift of $1.3 \AA$ and $0.6 \AA$. The second STIS spectrum has a continuum flux level 14\% larger and its absorption lines are blue shifted by only $0.4 \AA$. Based on the ephemeris of Ribiero \& Diaz (2007), the Table 1 STIS spectra extend in orbital phase from 0.67 to $0.81,0.33$ to 0.47 , and 0.35 to 0.49 respectively. The STIS spectra cover the interval from $1150 \AA$ to $1720 \AA$.

\subsection{The combined spectra}

The FUSE spectrum and the first STIS spectrum of V3885 Sgr are shown in Figure 1 before correction for reddening; the spectra are characterized by broad and deep absorption lines from the source (disk plus wind/chromosphere). These broad absorption lines are blue shifted by about $1.5 \AA$ and $2.5 \AA$ in the FUSE and STIS spectra respectively; they are about $4 \AA$ in width $(\sim 1,000 \mathrm{~km} / \mathrm{s})$ and exhibit the same asymmetry in both the FUSE and STIS spectra. The red edge of the absorption is steep, while the blue edge has a more gradual 
slope, resembling the blue-shifted absorption part of a P-Cygni profile. However, only the C IV (1550) and Nv (1240) lines exhibit a true P-Cygni profile, while all the other broad absorption lines do not. All these lines belong to high ionization species: N III, N IV, Nv, Si III, Si IV, C III, C IV, O vi, P v, and He II.

In addition to the broad lines, there are many narrow and deep absorption lines. They have a width varying between about $0.15 \AA$ (e.g. O I) and $0.3 \AA$ (for $\mathrm{HI}$ ) and are also blue shifted, but by a much smaller amount $(\sim 0.2 \AA)$ than the broad lines. Many of the narrow lines belong to low order ionization species; however, some also belong to higher ionization species. Some of the sharp lines are known to be interstellar, such as Ar I and Al II. For simplicity we put all these lines in the ISM category. Additional details concerning the STIS spectra are in Hartley et al. (2002). Table 2 and Table 3 list all the lines, separated into FUSE lines and STIS lines.

From the AAVSO web site 1 we determine that during the month of April 2000 and through May 2000, the V3885 Sgr visible magnitude varied by only 0.2 . The first of the Table 1 STIS spectra falls within this time frame and it is closest in time to the FUSE spectrum. Also, the orbital phases of the two spectra are nearly the same. Finally, the first STIS spectrum most closely matches the FUSE spectrum in the overlap region. All of the spectra have been corrected for interstellar reddening of $E(B-V)=0.02$ (§3). We choose the first STIS spectrum to combine with the FUSE spectrum for analysis. Figure 2 shows the combination of the FUSE spectrum with the three STIS spectra. The upper panel shows the overlap region. The FUSE spectrum is red, the first STIS spectrum is cyan, the second STIS spectrum is magenta, and the third STIS spectrum is brown. For optimum fit to the FUSE spectrum, the STIS spectra have been divided by $0.98,1.12$, and 0.94 respectively. The top panel shows the very good match of the spectra in the overlap region, while the lower panel shows that, when normalized, the three STIS spectra track each other with excellent fidelity. The STIS spectra are noisy in the overlap region while the upper panel of Figure 2 shows that the STIS and FUSE spectra continua superpose accurately. We produced a combined spectrum for analysis by terminating the STIS spectrum at $1181.5 \AA$ at the short wavelength end, dividing it by 0.98 , and adding the FUSE spectrum, beginning at $1181.5 \AA$ and extending to shorter wavelengths.

We suggest, without detailed analysis, that the differences among the STIS spectra could be explained by slight variation in the mass transfer rate from the secondary star. Whatever the cause of the variation, the fact that the normalized STIS spectra have the same SED simplifies calculation of a system model.

\footnotetext{
${ }^{1}$ www.aavso.org
} 


\section{Initially adopted system parameters}

Table 4 (and notes) lists initially adopted parameters and their sources. Several of the system parameters are poorly known. As mentioned earlier, the Hipparcos parallax is $9.11 \pm 1.95$ mas, which corresponds to a distance of $110 \pm 30$ pc. Knigge (2007) determines $M_{2}=0.475 M_{\odot}$ for $P=0.20716^{\mathrm{d}}$, and a mean $M_{\mathrm{wd}}=0.7 M_{\odot}$. We adopt both masses and note that the resulting $q=0.68$ is in agreement with the observationally determined $q \sim 0.7$ (Hartley et al. 2002). Panei, Althaus, \& Benvenuto (2000) lists a WD radius of $1.091 \times 10^{-2} R_{\odot}$ for a $0.70 M_{\odot}$ homogeneous zero temperature Hamada-Salpeter carbon model WD. In our subsequent study of the observed spectra we correct the radius for the adopted WD $T_{\text {eff }}$; the contribution of the WD to the system synthetic spectrum is appreciable. Tablenotes to Table 4 list alternative sources for the adopted initial parameters. Those notes do not include the determination of $i \sim 25^{\circ}-30^{\circ}$ (Cowley \& Crampton 1977). This determination was based on radial velocity observations with a large scatter; the derived velocity amplitude was $K=74 \mathrm{~km} \mathrm{~s}^{-1}$ while much more accurate data gives a value of $K=165 \mathrm{~km} \mathrm{~s}^{-1}$ (Hartley et al. 2005).

Verbunt (1987) determined a rough upper limit of $E(B-V)$ of 0.04 (and a preferred value of $E(B-V)=0.0$ ) for V3885 Sgr based on a study of the $2200 \AA$ "bump" in $I U E$ spectra. Bruch \& Engel (1994) list $E(B-V)=0.02$, which we adopt.

A number of studies have considered the tidal cutoff boundary, $r_{d}$, of accretion disks Paczynski (1977); Papaloizou \& Pringle (1977); Whitehurst (1988); Schwarzenberg-Czerny \& Różyczka (1988); Whitehurst \& King (1991); Goodman (1993)). These authors agree on $r_{d} \approx 0.33 D$, where $D$ is the separation of the stellar components. We adopt this expression for the tidal cutoff radius of the accretion disk.

\section{The analysis program: BINSYN}

Our analysis uses the program suite BINSYN (Linnell \& Hubeny 1996); recent papers (Linnell et al. 2007, 2008a) describe its application to CV systems in detail. Briefly, an initial calculation produces a set of annulus models for a given WD mass, radius, and mass transfer rate. This calculation uses the program TLUSTY 2 (Hubeny 1988; Hubeny \& Lanz 1995). The program considers the radial and vertical structure of the disk independently; the radial structure is based on the standard model (FKR) and so follows the prescribed relation between local $T_{\text {eff }}$ and the annulus radius. The vertical structure is solved, self-consistently,

\footnotetext{
${ }^{2}$ http://nova.astro.umd.edu
} 
as described by Hubeny (1990) and Hubeny \& Hubeny (1998). The set of annulus models covers the accretion disk from its innermost (WD) radius to just short of its outermost (tidal cutoff) radius.

The primary source of viscosity in CV accretion disks is MRI (Balbus \& Hawley 1991). Hirose, Krolik, \& Stone (2006) calculate MHD models with local dissipation of turbulence and show that the vertical extent of an annulus is greater than in previous models. Blaes et al. (2006) show that magnetic support has a significant effect on synthetic spectra of black hole $(\mathrm{BH})$ accretion disk annuli and illustrate the effect in the case of a BH system with $M_{\mathrm{BH}}=6.62 M_{\odot}$ and with an adopted $\alpha=0.02$. They are able to simulate the effect of magnetic support within the TLUSTY framework by adjusting the TLUSTY parameters $\zeta_{0}$ and $\zeta_{1}$ and use this simulation to calculate the effect on synthetic spectra. The primary effect is a hardening of the spectrum. King, Pringle,\& Stone (2007) point out that MHD models that produce viscosity via MRI require $\alpha$ values that are a factor 10 smaller than the $\alpha=0.1-0.4$ required by observational evidence and suggest that some caution still is needed in accepting the MHD results. Our models have used the default values $\zeta_{0}=\zeta_{1}=0.0$ and we assume the effect of magnetic support on our annulus spectra, with $M_{\mathrm{wd}}=0.7 M_{\odot}$, is insignificant.

Table 5 lists properties of annuli calculated with TLUSTY(v.202) for a mass transfer rate of $5.0 \times 10^{-9} M_{\odot} / \mathrm{yr}^{-1}$. This value initially was chosen for test purposes because of the similarity of V3885 Sgr to IX Vel (Linnell et al. 2007); see $§ 5$ for a detailed discussion. The control file to calculate a given annulus requires a radius of the WD in units of $R_{\odot}$. All of the annuli used the radius of a zero temperature WD for a homogeneous carbon HamadaSalpeter $0.70 M_{\odot}$ model from Panei, Althaus, \& Benvenuto (2000). All of the annuli are solar composition H-He models, and the models through $r / r_{\mathrm{wd}, 0}=20.00$ are converged LTE models. The remaining annuli are LTE-grey models (see the TLUSTY Users Guide for an explanation). A Shakura \& Sunvaev (1973) viscosity parameter $\alpha=0.1$ was used in calculating all annuli. Each line in the table represents a separate annulus. Temperatures are in Kelvins. The column headed by $m_{0}$ is the column mass, in $\mathrm{gm}^{-2}$, above the central plane. The columns headed by $z_{0}$ and $N_{e}$ are, respectively, the height $(\mathrm{cm})$ above the central plane for a Rosseland optical depth of $\approx 0.7$ and the electron density $\left(\mathrm{cm}^{-3}\right)$ at the same level. The column headed by $\log g$ is the $\log$ gravity ( $\operatorname{cgs}$ units) in the $z$ direction at a Rosseland optical depth of $\approx 0.7$. The column headed by $\tau_{\text {Ross }}$ is the Rosseland optical depth at the central plane. We call attention to the fact that the annuli are optically thick to the outer radius of the accretion disk. 
Following calculation of annulus models for assigned $M_{\mathrm{wd}}$ and $\dot{M}$, program SYNSPEC(v.48) 3 (Hubeny, Lanz, \& Jeffery 1994) is used to produce a synthetic spectrum for each annulus. We adopted solar composition for all synthetic spectra. The synthetic spectra include contributions from the first 30 atomic species, assumed to be in LTE.

We set SYNSPEC to produce light intensities at 10 zenith angles (equally spaced in $\cos (\gamma)$ between 1.0 and 0.1 , where $\gamma$ is the angle between the given direction and the local normal) at each calculated wavelength for all the individual annulus synthetic spectra. This choice automatically accounts for wavelength-dependent limb darkening in calculating synthetic spectra at different orbital inclinations. BINSYN calculates a synthetic spectrum for each of the annuli specified in the BINSYN system model by interpolation among the array of TLUSTY annulus models, with proper allowance for inclination.

BINSYN models the complete CV system, including the WD, secondary star, accretion disk face, and accretion disk rim as separate entities. The model represents phase-dependent and inclination-dependent effects, including eclipse effects and irradiation effects, on all of the system objects. The representation of the stars requires polar $T_{\text {eff }}$ values and gravitydarkening exponents. BINSYN represents the accretion disk by a specified number of annuli, where that number typically is larger than the number of TLUSTY annulus models. The accretion disk $T_{\text {eff }}$ profile may follow the standard model, but, alternatively, the profile may be specified by a separate input file.

BINSYN has provision to represent a bright spot on the rim face, but the low amplitude $V$ light curve by Ribiero \& Diaz (2007) (Fig. 10) is consistent with light variation of an irradiated secondary star so we do not include a rim bright spot. Based on the analysis by Smak (2002) we set the rim $T_{\text {eff }}$ equal to the $T_{\text {eff }}$ of the outermost annulus.

\section{A V3885 Sgr system model}

We adopted system parameters listed in Table 4 for an initial test. Based on the similarity to IX Vel (comparable periods, visual magnitudes, parallaxes, CV class, spectroscopic properties (Hartley et al. 2002)), we first tried a standard model accretion disk with the same $\dot{M}, 5.0 \times 10^{-9} M_{\odot} \mathrm{yr}^{-1}$, as for IX Vel (Linnell et al. 2007), and, as for IX Vel, the (initial) model included a 60,000K WD. In constructing a model accretion disk it is necessary to allow for the change in WD radius from the zero temperature model. We used Table 4a of Panei, Althaus, \& Benvenuto (2000) to estimate the $60,000 \mathrm{~K}$ WD radius at $0.0134 R_{\odot}$.

\footnotetext{
${ }^{3}$ http://nova.astro.umd.edu
} 
Our BINSYN model specified 33 annuli for the accretion disk, with assigned radii and corresponding standard model $T_{\text {eff }}$ values listed in Table 6 . The larger hot WD radius than a zero temperature WD radius at a given multiple of the 'WD radius' produces a lower Table 6 temperature than Table 5 (e.g., at the $r / r_{\text {wd }}$ value 6.0 in column 1 of both tables). The synthetic spectrum for a given BINSYN annulus is calculated by interpolation (temperature-wise) among the Table 5 entries. Ideally, we could recalculate the whole series of annulus models (via TLUSTY) based on the new value of $R_{\mathrm{wd}}$, but the change in the individual annulus models would be very small and would not be warranted in view of other uncertainties (e.g., the system parallax).

Table 5 ends at a $T_{\text {eff }}$ of $7711 \mathrm{~K}$. TLUSTY fails for larger radii, even in the gray model case. The net effect is that the calculated BINSYN outer annuli have synthetic spectra corresponding to $7711 \mathrm{~K}$ even if the specified standard model calls for a lower temperature. The effect on the synthetic spectrum of failing to follow the standard model is negligible. Adding a lower temperature $(3500 \mathrm{~K})$ stellar synthetic spectrum to the array of Table 5 , thereby permitting interpolation to a lower temperature in the outer BINSYN annuli, made no detectable change in the system synthetic spectrum. As separate issues, the outer annuli temperatures should not fall below 6000K to avoid outbursts (Osaki 1996; Lasota 2001) and tidal dissipation is expected to raise the outer accretion disk temperature above the standard model value (Smak 2002).

The overall SED fit to the combined spectrum of $\S 2.3$, using initial parameters, and judged by visual estimate, was very good with a standard model accretion disk temperature profile. This was in contrast to IX Vel which required modification of the temperature profile from a standard model to achieve an acceptable fit (Linnell et al. 2007). Assessing the quality of fit for V3885 Sgr is complicated by the presence of strong absorption features of highly ionized material (Figure 1), probably associated with a wind (Hartley et al. 2002). At this initial stage, the extreme synthetic spectrum FUV region had slightly too large flux: the only parameter (either $\dot{M}$ or $T_{\text {eff,wd }}$ ) available to adjust the flux while preserving the fixed normalizing factor was the WD $T_{\text {eff }}$. $(\dot{M}$ could be adjusted but that route produced detectably larger residuals longward of $1500 \AA$. Small adjustments of $\dot{M}$ move the entire synthetic spectrum up or down without appreciable change in the spectral gradient. Similarly, a change in $i$ does not change the spectral gradient. We consider a boundary layer in $\S 6$.) We reduced the WD $T_{\text {eff }}$ to $57,000 \mathrm{~K}$ and found this produced an optimum fit as judged by eye estimate. The new model neglected the very small change in WD radius between $T_{\text {eff }}=60,000 \mathrm{~K}$ and $57,000 \mathrm{~K}$, which would have affected the tabular radii in Table 6 .

Figure 3 shows the FUV fit to the combined FUSE and STIS spectra, dereddened for $E(B-V)=0.02$. We call attention to the broad absorption lines which we attribute to a 
wind/chromosphere and that affect a major part of the FUSE spectrum. Note the deep ISM Lyman series lines, the broad and deep absorption by OI and NIII near $990 \AA$, the deep and possible multi-component Ly $\beta$ line near $1025 \AA$, and the broad lines of SiIII, PV, SIV and SiIV in the $1110 \AA$ to $1140 \AA$ interval. The overlapping and possibly multicomponent lines depress the apparent continuum over the entire interval from $970 \AA$ to $1170 \AA$.

The normalizing factor to divide the synthetic spectrum to superpose it on the observed, dereddened combined FUSE and STIS spectrum, corresponding to an exact distance of 110 $\mathrm{pc}$, is $1.1473 \times 10^{41} \mathrm{~cm}^{2}$. We stress that this is not an adjustable parameter. In $\S 5.1$ we consider the effects of varying the 'exact' distance corresponding to $\pm 1 \sigma$ in the Hipparcos parallax. The spectrum at the bottom is the contribution of a $57,000 \mathrm{~K}$ WD. For simplicity we assume the WD is rotating synchronously; we do not fit any observed absorption lines in detail. The program assumes that the accretion disk blocks the view of the lower half of the WD. In addition, BINSYN allows for the partial visibility of that part of the WD not obscured by the accretion disk and above the observer's horizon, and includes wavelength-dependent limb darkening. The spectral resolution of all the synthetic spectra is $0.02 \AA$ (§5.2.). The adopted orbital phase for the synthetic spectra was 0.75 , in agreement with the observed spectra, §2. Adopting an orbital phase of 0.25 produced a clear misalignment of the calculated $960 \AA$ peak with the observed one.

We check the apparent best fit, by eye estimate, with a reduced chi squared $\left(\widetilde{\chi}^{2}\right)$ test. We did not smooth the observed spectrum for this test. The presence of unmodeled wind/chromosphere features in the observations produces large residuals (especially in the FUV, Figure 3). A $\tilde{\chi}^{2}$ test of our model fit to the observed continuum requires masking those spectral regions excluded from the fit (i.e., the wind/chromosphere features). We initially masked spectral lines by hand, including the deep Lyman lines. (The Lyman lines in the model, mostly from the accretion disk, are washed out by Keplerian rotation.) This first stage masking produced a major reduction (from 154.39 to 7.38 ) in $\widetilde{\chi}^{2}$ as compared with the unmasked spectrum. There remained a major "valley" in a residuals plot, described in the following paragraph, with the largest (negative) residuals near $1100 \AA$. Our previous discussion has identified this effect with overlapping absorption features from a wind/chromosphere which depresses the entire spectral interval over the approximate range $970 \AA$ to $1170 \AA$. BINSYN has no present capability to model a wind/chromosphere and further progress requires masking the affected spectral interval. We therefore masked the entire region from $970 \AA$ to $1170 \AA$, producing a further large reduction in $\widetilde{\chi}^{2}$ (from 7.38 to 2.65 ).

Figure 4 shows plots of the residuals in the three cases. The top plot, displaced upward by 12.0 ordinate units, is the residuals for the unmasked case. The middle plot, displaced upward by 4.0 ordinate units, is the partially masked case. The lower (undisplaced) plot 
is the final set of residuals adopted for this study. In each case a horizontal line marks the undisplaced reference. The change from the top to middle plot mostly results from masking of individual spectral features, such as the Lyman lines, the CIV $\lambda 1548$ feature, etc. The STIS spectrum appears to have a defect beginning at $1710 \AA$, and the three STIS spectra show more variability longward of the HeII $\lambda 1640$ line than in other spectral regions. We terminated our calculated fit just longward of the HeII $\lambda 1640$ line. The middle plot clearly shows the residual depression after individual spectral features have been masked and the bottom plot shows the overall improvement in residuals after masking the $970 \AA$ to $1170 \AA$ region. The improvement is even more apparent on individual plots with extended ordinate axes.

The lower plot still has fairly large outliers. Although further selective masking could be done, we consider it dangerous to go very far in suppressing observations that disagree with the model. Residual absorption features could be masked but there are positive outliers that then would bias the solution. We anticipate that it will be possible to locate an optimum model with the chosen masked set of observations. The final masked version of the observed combined spectra includes 4332 tabulated wavelengths and corresponding flux values.

It is particularly unfortunate for this analysis that most of the FUSE spectrum must be masked, but it is unavoidable: the masked region clearly is strongly affected by the broad and deep absorption lines of high excitation species as described in 2.3 . The high excitation involved excludes the lines from production by the accretion disk itself or the WD. Further, ignoring the high excitation aspect for the moment, it would not be possible to simulate the depressed $970 \AA-1170 \AA$ spectral interval by adopting a modified accretion disk $T_{\text {eff }}$ profile. Our experience with non-standard model accretion disks is that the calculated SED profile can have its slope changed over an extended spectral region but it is not possible to put a "hole" in the SED.

We calculated $\widetilde{\chi}^{2}$ values for a grid of 34 models in the $\dot{M}, T_{\text {eff,wd }}$ plane, centered on the best visual estimate model fit to the masked observations. Table 7 lists those values plus $\widetilde{\chi}^{2}$ values for 4 additional $i$ values (to be discussed separately). The aim is to provide a sufficiently extended grid that a subset of $\widetilde{\chi}^{2}$ values define a $\widetilde{\chi}^{2}$ contour that encloses the region of minimum $\widetilde{\chi}^{2}$, given the adopted values of other parameters. Calculation of a given model is a time-consuming process and a compromise is necessary between total computational time and density of grid points. Our computed grid is sparse but our judgment is that it meets our needs, given the uncertainties of separately adopted parameters. We have used the IDL routine CONTOUR to produce contour plots for the Table $7 i=65^{\circ}$ values. The $\dot{M}=5.0 \times 10^{-9} M_{\odot} \mathrm{yr}^{-1}, T_{\text {eff,wd }}=57,000 \mathrm{~K}$ model has the smallest tabular $\tilde{\chi}^{2}$ value and we take that model to be our solution for V3885 Sgr. We discuss this choice and 
the topic of grid density, including its adequacy, in more detail in $\S 6$.

We have sampled the $\dot{M}, T_{\text {eff,wd }}$ plane for the case $M_{\text {wd }}=0.8 M_{\odot}$ with two new models: $\dot{M}=5.0 \times 10^{-9} M_{\odot} \mathrm{yr}^{-1}$ and $T_{\text {eff,wd }}=57,000 \mathrm{~K}$, producing $\widetilde{\chi}^{2}=3.06$, and $\dot{M}=$ $5.0 \times 10^{-9} M_{\odot} \mathrm{yr}^{-1}$ and $T_{\text {eff,wd }}=52,000 \mathrm{~K}$, producing $\widetilde{\chi}^{2}=3.81$. The corresponding $\widetilde{\chi}^{2}$ values from Table 7 are 2.65 and 3.39 respectively. A preferred value of $M_{\mathrm{wd}}$ is not possible by $\widetilde{\chi}^{2}$ analysis of the existing data. The SED accretion disk model is not a strong function of $M_{\mathrm{wd}}$ for the change from $0.7 M_{\odot}$ to $0.8 M_{\odot}$. Said differently, Figure 5 remains roughly constant, with a small systematic shift for changes in $M_{\mathrm{wd}}$ of about 0.1 or less.

An improved value of $M_{\mathrm{wd}}$ may be possible with further optical region spectroscopy. Ribiero \& Diaz (2007) find that the $\mathrm{H} \alpha$ emission line arises from irradiaton of the secondary while the (doubled) $\lambda 6678 \mathrm{HeI}$ emission line associates with the accretion disk. If an accretion disk model that produces secondary component emission lines were available, a fit to the HeI line could constrain $i$.

Table 7 includes 4 models for different assumed values of $i$ and with other parameters the same as the preferred model. There is a very sharp minimum of $\widetilde{\chi}^{2}$ at the adopted $i=65^{\circ}$. At this inclination there is an eclipse of the outer accretion disk at orbital phase 0.0 with photometric effects much smaller than the predicted variation due to irradiation of the secondary. Increasing the inclination to $70^{\circ}$ greatly deepens the depth of accretion disk eclipse and barely fails to eclipse the WD; it would produce light curve effects that are not observed (Ribiero \& Diaz 2007, Figure 10). However the observed light variation is complex and may include a contribution from a rim bright spot. An inclination of $71^{\circ}$ would eclipse the WD and produce a major light curve variation. It is not possible to use the $\widetilde{\chi}^{2}$ $i$-dependent variation to determine $i$. The calculated system flux is a strong function of $i$ because of the changing projection of the flux-dominating accretion disk on the plane of the sky. The fit of the model spectrum to the observations requires a normalizing factor that is given by the Hipparcos parallax. The sharp minimum of $\widetilde{\chi}^{2}$ for $i=65^{\circ}$ results from the joint consistency of that parallax and the adopted $i$ value. Actual determination of $i$ would require observational effects intrinsic to the system, i.e., eclipses.

Until a model that includes simulation of the wind/chromosphere is available it is doubtful that substantial further reduction in $\widetilde{\chi}^{2}$ can be achieved. Based only on the $\widetilde{\chi}^{2}$ plot, Figure 5 , we estimate the parameter errors in $\dot{M}, T_{\text {eff,wd }}$, and $i$ as $2.0 \times 10^{-10} M_{\odot} \mathrm{yr}^{-1}, 3000 \mathrm{~K}$, and, from Table 7, $2.0^{\circ}$ respectively. However, shifts in $\dot{M}$ to match the uncertainty in the Hipparcos parallax (§5.1.) require a larger $\dot{M}$ error of $2.0 \times 10^{-9} M_{\odot} \mathrm{yr}^{-1}$ which we adopt for the system solution. Further, in recognition of the sparse grid of models and their associated $\widetilde{\chi}^{2}$ values, we increase the estimated $T_{\text {eff,wd }}$ error to $5000 \mathrm{~K}$. 
Our final system parameters are in Table 8. Where appropriate we have provided estimated error limits. Figure 6 shows the final model compared with the combined observations (not the masked observations). The blue line is the contribution of the $57,000 \mathrm{~K}$ WD. The lower of the two black lines is the contribution of the accretion disk, and the upper black line is the system model.

\subsection{The effects of Hipparcos parallax uncertainty}

It is possible to test the effect of uncertainty in the Hipparcos parallax by changing the normalizing divisor for superposing the synthetic spectrum on the combined FUSE and STIS spectra. Changing the parallax by $1 \sigma$ to 7.16 mas changes the normalizing divisor from $1.147 \times 10^{41} \mathrm{~cm}^{2}$ to $1.857 \times 10^{41} \mathrm{~cm}^{2}$. The synthetic spectrum is displaced downward by approximately two flux units on Figure 3 (the $1000 \AA$ peak moves from an ordinate value of 10. to 8.) Compensating for the change would require increasing $\dot{M}$ to perhaps 6.5

or $7.0 \times 10^{-9} M_{\odot} \mathrm{yr}^{-1}$. Correspondingly, changing the parallax to 11.06 mas changes the normalizing divisor to $7.784 \times 10^{40} \mathrm{~cm}^{2}$ and displaces the synthetic spectrum upward by about two flux units, from a $1000 \AA$ peak of 10 to 12 . Compensating would require decreasing $\dot{M}$ to perhaps 4.0 or $4.5 \times 10^{-9} M_{\odot} \mathrm{yr}^{-1}$.

\subsection{Correction for the ISM}

Recent papers have included corrections for line absorption by the ISM (Godon et al. 2008; Linnell et al. 2008a). A custom spectral fitting package is used to estimate the temperature and density of the interstellar absorption lines of atomic and molecular hydrogen. The ISM model assumes that the temperature, bulk velocity, and turbulent velocity of the medium are the same for all atomic and molecular species, whereas the densities of atomic and molecular hydrogen, and the ratios of deuterium to hydrogen and metals (including helium) to hydrogen are adjustable parameters. The model uses atomic data of Morton (2000, 2003 ) and molecular data of Abgrall (2000). The molecular hydrogen transmission values have been checked against those of McCandliss (2003). The calculation requires fairly high spectral resolution and we have calculated all of the TLUSTY-SYNSPEC synthetic spectra at a resolution of $0.02 \AA$; we used the same resolution in calculating the system synthetic spectra. The ISM parameters are in Table 9. Figure 7 shows the corrected version of part of Figure 6. The ISM model produces a good representation of the observed deep cores of the Lyman series lines and the Lyman cutoff, as well as some lines in other spectral regions and clearly shows that the ISM produces the observed Lyman lines. The broad, high exci- 
tation absorption regions from the accretion disk corona, chromosphere, or wind, identified in Table 2 and Table 3, remain as prominent residuals. As stated above, the Lyman lines were masked in the fit to the model and the spectral region shortward of the Lyman limit was masked so only the Lyman region continuum participated in the model solution. This was proper since a fit to the ISM does not affect the choice of the model parameters listed in Table 8. There are small positive residuals from the model near the short wavelength end. This could be interpreted as evidence for an unmodeled very high temperature contribution, discussed in the following section.

\section{Discussion}

In a well-known paper Wade (1988) showed that neither steady state model accretion disks based on Planck functions or stellar model atmospheres could simultaneously fit the colors and absolute luminosities of a set of NL systems (V3885 Sgr was included in the study). Tomographic analyses of accretion disks (Rutten, van Paradijs, \& Tinbergen 1992) show clear departures from standard model temperature profiles (FKR). Recent analyses of the NL systems MV Lyr, IX Vel, QU Car, and UX UMa (Linnell et al. 2005, 2007, 2008a, b) showed, in each case, that a standard model accretion disk synthetic spectrum could not accurately fit observed spectra. These results suggest that physical conditions in possibly all NL systems are so complex, with winds, coronae, iron curtains, etc., that an accurate fit by a standard model synthetic spectrum is too much to expect. Consequently, the most interesting result of this study is that a standard model synthetic spectrum plus WD accurately fits the observations $\left(\widetilde{\chi}^{2}=2.65\right)$ after masking the effects of a wind/chromosphere. This conclusion is particularly true in light of the fact that the system distance is known so the fit is in absolute flux units. It is worth emphasizing that the model presented here includes a measurable contribution from a hot source, in addition to the accretion disk, sufficiently large that limits can be set on the hot source $T_{\text {eff }}$. Assuming that the hot source is a WD (see below), this is the first time the effective temperature of a hot WD has been constrained during the high brightness state of a nova-like variable.

As discussed by FKR, half of the potential energy liberated in the fall of the mass transfer stream from the L1 point (essentially from infinity) to the WD surface appears as radiated energy. Energy conservation requires an accounting for the other half and the presence of a hot boundary layer (BL) is a common prescription. The problem is that BLs with the prescribed properties are not observed (Córdova 1995). The absence of an observed but predicted (FKR) BL in high $\dot{M}$ CV systems has an extensive history: (Ferland 1982; Kallman \& Jensen 1985; Patterson \& Ravmond 1985; Hoare \& Drew 1991, 1993; Vrtilek et al. 1994; Idan \& Shaviv 
1996). One proposed explanation is that the WD is rotating close to Keplerian rotation, with no BL predicted. However, HST measurements starting with Sion et al. (1994) have measured rotational WD velocities too low to explain "missing" BLs. An alternative explanatory theme is that the absence of an observed BL associates with a wind, and in V3885 Sgr a wind is clearly present (Hartley et al. 2002, 2005).

BINSYN has an option to simulate a BL by specifying the innermost annulus radial thickness and $T_{\text {eff }}$. We applied the theory of Godon, Regev, \& Shaviv (1995) to obtain those two parameters. This theory derives a BL with a $T_{\text {eff }} \sim 100,000 \mathrm{~K}$ and radial thickness of about $0.1 R_{\mathrm{wd}}$. This BL $T_{\text {eff }}$ is consistent with a value from wind ionization (Hoare \& Drew 1991) and the theory of Idan \& Shaviv (1996). The Godon, Regev, \& Shaviv (1995) model adopted $M_{\mathrm{wd}}=1.0 M_{\odot}$ and $T_{\mathrm{wd}, \mathrm{eff}}=20,000 \mathrm{~K}$. Addition of the BL made a nearly negligible change in our system SED. There was a barely detectable increase in the system flux at the short wavelength limit over the SED of our original model, producing a slightly better fit in that spectral region. The evidence in favor of a BL is inconclusive; a model including a $100,000 \mathrm{~K}$ BL and a $57,000 \mathrm{~K}$ WD is consistent with the observations. Popham \& Naravan (1995) calculate BL models and illustrate their theory for the case $\dot{M}=10^{-8} M_{\odot} \mathrm{yr}^{-1}$ and zero WD rotation, leading to a somewhat higher BL $T_{\text {eff }}$. In a discussion among the coauthors Godon noted that the BL $T_{\text {eff }}$ might be as high as $200,000 \mathrm{~K}$ but probably would not be visible at $i=65^{\circ}$ because of an inflated inner disk; an additional reason for not including a BL. A model including the $100,000 \mathrm{~K}$ BL but with our WD contribution suppressed (i.e., requiring the hot source to be the $\mathrm{BL}$ exclusively) would be strongly inconsistent with the observations. We are unaware of any BL models that treat MHD effects.

Is it possible to exclude a different source for the WD contribution of our present model? We seek some spectral feature that could distinguish the WD contribution from a different prospective source. In Figure 6 the WD Ly $\gamma$ absorption line appears to make an important contribution to the system synthetic spectrum. A different smoothly varying source, such as a black body (BB) or power law source might produce a much poorer fit to the observed Ly $\gamma$ wings and so be excludable. We tested this possibility by replacing the WD synthetic spectrum by a $57,000 \mathrm{~K}$ BB with the same size as the replaced WD. The existing model uses light intensities at 10 zenith angles (§4), produced by SYNSPEC, for each annulus. Use of the BB spectrum required a change to flux-based synthetic spectra with an adopted wavelength-independent limb darkening coefficient; we chose 0.6. The system synthetic spectrum SED was essentially unchanged but required a slight adjustment of the normalizing factor to reproduce the same fit as with the original model, likely a result of the change to a wavelength-independent limb darkening coefficient. The fit at the short wavelength limit was slightly better, but it is uncertain whether this arises from the BB replacement or the change in limb darkening. In any event, the Ly $\gamma$ line was slightly shallower in the new 
simulation, arguing weakly in favor of the presence of a WD, but the fit to the wings was essentially the same. The proposed spectral feature test fails and we are unable to exclude a $\mathrm{BB}$ as the source of the required hot addition to the accretion disk. The SED of a power law source would differ from that of a BB source and should produce a poorer fit to the observed spectrum; without explicit test we discount this type source as a reasonable candidate.

To answer the question "What is the source of the hot addition to the accretion disk?" the most reasonable working hypothesis is that the hot source is a WD. A WD is known to be present (it is a CV system) and the inclination, $i$, is such that the WD should be visible. A spectral source substitute for a WD (e.g., BB) would need not only to explain why the WD spectrum is not seen but also why a BB source of the same $T_{\text {eff }}$ and size (which sets the radii of the accretion disk annuli and thus their $T_{\text {eff }}$ values (Table 6)) and physical location (exactly centered on the accretion disk) is a reasonable substitute.

The adequacy of our set of 34 models in populating the $\widetilde{\chi}^{2}\left(\dot{M}, T_{\text {eff,wd }}\right)$ plane requires justification At issue is the question "How dense a grid is adequate?" Phrased differently, "As we produce a denser and denser grid, and keeping in mind the committment of computer time, how do we know when to stop?" A very important point is that our solution adopts values for the parameters $\mathrm{M}(\mathrm{wd}), \mathrm{M}(\mathrm{sec})$, Period, $i$, parallax, and $E(B-V)$. A solution with a different value of any one of these would require recalculation of an entire new plane of $\widetilde{\chi}^{2}\left(\dot{M}, T_{\text {eff,wd }}\right)$ values, like Figure 5 , and search of it for the minimum $\widetilde{\chi}^{2}$. That process would be required, for example, if we study the implications of changing the adopted parallax by one standard error. The solution we have found from our set of $\widetilde{\chi}^{2}$ values is valid only under the assumption that the other fixed parameter values are accurate.

Our Table 7 values for a specific $\dot{M}$ and variable $T_{\text {eff,wd }}$, or specific $T_{\text {eff,wd }}$ and variable $\dot{M}$, extend to a $\widetilde{\chi}^{2}$, when rounded to the nearest half integer, of at least 4.0. We consider that value to be enough larger than the minimum of 2.65, and the resolution in $\dot{M}$ and $T_{\text {eff,wd }}$ sufficiently fine, to provide reasonable assurance that the (ideal) global minimum has been encircled. Figure 5, produced with IDL routine CONTOUR, presents contours of constant $\widetilde{\chi}^{2}$ including closed contours that encircle the tabular minimum.

We reiterate the point that possibly our most interesting result is the accurate fit to a standard model. We note that a visual estimate ("chi by eye") selected the best model.

Hartley et al. (2002) discuss the wind in V3885 Sgr and point to the lack of correlation between wind activity and system luminosity, an inconsistency with expectation for line-driven winds. The theory of line-driven winds has been applied to CV systems by Perevra. Hillier. \& Turnshek (2006) and Proga (2003a, b). See the review by Drew \& Proga (2000). Proga (2003a) considers both radiation-driven and MHD effects and shows that hy- 
brid models that include both MHD and line-driving produce larger $\dot{M}_{\text {wind }}$ values, of order $10^{-10} M_{\odot} \mathrm{yr}^{-1}$, but Proga (2003b) shows that a pure line-driven wind produces an improved

fit to observed line shapes but with a lower $\dot{M}_{\text {wind }}$. As Hartley et al. (2002) point out, the lack of correlation between wind activity and system luminosity may argue in favor of MHD effects. In either case, the theoretically derived values of $\dot{M}_{\text {wind }}$ are far smaller than the mass transfer $\dot{M}$ and essentially all of the mass transfer stream must be deposited on the WD.

Using eq. 2 of Sion (1995), the $T_{\text {eff }}$ of a compressionally-heated WD is given by $T_{\text {eff }}=$ $\left[\left(f G M_{\mathrm{wd}} \dot{M}\right) /\left(4 \pi R_{\mathrm{wd}}^{3} \sigma\right)\right]^{0.25}$, where $f$ is the fraction of the accretion energy applied to compressional heating and is in the range $0.15-0.20$. For $f=0.15$ and the parameters of Table 8 we calculate $T_{\text {eff }}=52,500 \mathrm{~K}$, while for $f=0.20$ we find $T_{\text {eff }}=56,414 \mathrm{~K}$, well within the estimated error limit of our $57,000 \mathrm{~K}$ WD.

This study raises an obvious question: Why does a standard model accretion disk represent V3885 Sgr accurately while other apparently comparable systems violate the standard model? To rephrase the question: What equation or equations defining the standard model do the nonconforming systems violate? The standard model equations (e.g., FKR eq. 5.68) adopt axial symmetry and hydrostatic equilibrium, avoid MHD considerations (the MRI (Balbus \& Hawley 1991) source of viscosity), and assume a constant $\alpha$ for the entire accretion disk, corresponding to a constant local $\dot{M}$ within the accretion disk. The observed presence of spiral waves in V3885 Sgr (Hartley et al. 2005) violates the axial symmetry condition even in a system that fits the standard model. The impact of the mass transfer stream produces a departure from axial symmetry. These phenomena illustrate the point that the standard model is a simplified but very useful idealization. Just as weather in the Earth's atmosphere, from physically complex phenomena, produces transient but extended local departures from the ideally accurate hydrostatic equilibrium, we speculate that transients of unknown but physically complex origin may occur in localized regions of accretion disks that perturb the radiation characteristics from the standard model average. IUE spectra of UX Ursae Majoris show substantial time-dependent SED changes for that NL system (Linnell et al. 2008b). The adherence of V3885 Sgr to the standard model in this study may indicate an infrequently violated average. On the other hand, the observation times of nonconforming systems may occur at times when transients are prevalent but detailed frequent monitoring might discover times when their SEDs fit the standard model.

\section{Summary}

We use the Knigge (2007) period-secondary mass relation to assign the secondary mass and the mean WD mass from the same paper, consistent with an observationally-determined 
mass ratio, to set the V3885 Sgr WD mass. Based on a $\widetilde{\chi}^{2}$ analysis using 38 system models and corresponding synthetic spectra, we find that a standard model accretion disk with $\dot{M}=5.0 \pm 2.0 \times 10^{-9} M_{\odot} \mathrm{yr}^{-1}$, a $57,000 \pm 5000 \mathrm{~K} 0.7 M_{\odot} \mathrm{WD}$, and an orbital inclination of $65 \pm 2^{\circ}$ provide a system synthetic spectrum which optimally fits observed FUSE and STIS spectra of V3885 Sgr. The result is robust for small changes in the assumed $M_{\mathrm{wd}}$. A Hipparcos parallax fixes the divisor enabling the system synthetic spectrum to be matched to the observed spectra and establishes the fit on an absolute flux basis. The model thus provides realistic constraints on $\dot{M}$ and $T_{\text {eff }}$ for a large $\dot{M}$ system above the period gap.

We are grateful to the referee for a careful reading of the paper and for a series of comments that led to a significant improvement of the paper. P.S. acknowledges support from FUSE grant NNG04GC97G and HST grant GO-09724. Support for this work was provided by NASA through grant number HST-AR-10657.01-A to Villanova University (P. Godon) from the Space Telescope Science Institute, which is operated by the Association of Universities for Research in Astronomy, Incorporated, under NASA contact NAS5-26555. Additional support for this work was provided by the National Aeronautics and Space Administration (NASA) under Grant number NNX08AJ39G issued through the office of Astrophysics Data Analysis Program (ADP) to Villanova University (P. Godon). Participation by E. M. Sion, P. Godon and A. Linnell was also supported in part by NSF grant AST0807892 to Villanova University. This research was partly based on observations made with the NASA-CNESCSA Far Ultraviolet Spectroscopic Explorer. FUSE is operated for NASA by the Johns Hopkins University under NASA contract NAS5-32958.

\section{REFERENCES}

Abgrall, H., Roueff, E. \& Drira, I. 2000, A\&AS, 141, 297

Balbus, S. A., \& Hawley, J. F. 1991, ApJ, 376, 214

Blaes, O. M., Davis, S. W., Hirose, S., Krolik, J. H., \& Stone, J.M. 2006, ApJ, 645, 1402

Bruch, A. \& Engel, A. 1994, A\&AS, 1994, 104, 79

Cowley, A. P. \& Crampton, D. 1977, PASP, 89, 716

Córdova, F. A-D 1995, in X-Ray Binaries ed. W. H. G. Lewin, J. van Paradijs, E. van den Heuvel (Cambridge: Univ. Press)

Dixon, W.V., et al. 2007, PASP, 119, 527

Drew, J. E. \& Proga, D. 2000, New A Rev., 44, 21 
Ferland, G. J., et al. 1982, ApJ, 262, L53

Frank, J., King, A. \& Raine, D. 1992, Accretion Power in Astrophysics (Cambridge: Univ. Press) FKR

Godon, P., Regev, O., \& Shaviv, G. 1995, MNRAS, 275, 1093

Godon, P., \& Sion, E. M. 2002, ApJ, 566, 1084

Godon,P., Sion, E. M., Barrett, P. E., Hubeny, I., Linnell, A. P., \& Szkody, P. 2008, ApJ, 679,2008

Goodman, J. 1993, ApJ, 406, 596

Guinan, E. F. \& Sion, E. M. 1982, ApJ, 258, 217

Hartley, L. E., Drew, J. E., Long, K. S., Knigge, C., \& Proga, D. 2002, MNRAS, 332, 127

Hartley, L. E., Murray, J. R., Drew, J. E. \& Long, K. S. 2005, MNRAS, 363, 285

Haug, K. \& Dreschel, H. 1985, A\&A, 151, 157

Hirose, S., Krolik, J. H., \& Stone, J. M. 2006, ApJ, 640, 901

Hoare, M. G. \& Drew, J. E. 1991, MNRAS, 249, 452

Hoare, M. G., \& Drew, J. E. 1993, MNRAS, 260, 647

Howell, S. B., Nelson, L. A., \& Rappaport, S. 2001, ApJ, 550, 897

Hubeny, I. 1988, Comp. Phys. Comm., 52, 103

Hubeny, I. 1990, ApJ, 351, 632

Hubeny, I., Lanz, T., \& Jeffery, C. S. 1994, in Newsletter on Analysis of Astronomical Spectra No. 20, ed. C. S. Jeffery (CCP7;St. Andrews: St. Andrews Univ.), 30

Hubeny, I., \& Lanz, T. 1995, ApJ, 439, 875

Hubeny, I., \& Hubeny, V. 1998, ApJ, 505, 558

Idan, I., \& Shaviv, G. 1996, MNRAS, 281, 604

Kallman, T. R., \& Jensen, K. A. 1985, ApJ, 299, 277

King, A. R., Pringle, J. E., \& Livio, M. 2007, MNRAS, 376, 1740 
Knigge, C. 2007, MNRAS, 382, 1982

Lasota, J.-P. 2001, New Astronomy Reviews, 45, 449

Linnell, A. P., \& Hubeny, I. 1996, ApJ, 471, 958

Linnell, A. P., Szkody, P., Gänsicke, B., Long, K. S., Sion, E. M., Hoard, D. W., \& Hubeny, I. 2005, ApJ, 624, 923

Linnell, A. P., Godon, P., Hubeny, I., Sion, E. M. \& Szkody, P. 2007, ApJ, 662, 1204

Linnell, A. P., Godon, P., Hubeny, I., Sion, E. M., Szkody, P., \& Barrett, P. E. 2008a, ApJ, 676,1226

Linnell, A. P., Godon, P., Hubeny, I., Sion, E. M., \& Szkody, P. 2008b, ApJ, 688, 568

McCandliss, S. R. 2003, PASP, 624, 651

Metz, K. 1989, IBVS3385

Morton, D. C. 2000, ApJS, 149, 205

Morton, D. C. 2003, ApJS, 149, 205

Osaki, Y. 1996, PASP, 108, 39

Paczynski, B. 1977, ApJ, 216, 822

Panei, J. A., Althaus, L. G., \& Benvenuto, O. G. 2000, A\&A, 353, 970

Papaloizou, J., \& Pringle, J. E. 1977, MNRAS, 181, 441

Patterson, J., \& Raymond, J. C. 1985, ApJ, 292, 550

Pereyra, N. A., Hillier, D. J., \& Turnshek, D. A. 2006, ApJ, 636, 411

Perryman,M. A. C. et.al. 1997, A\&A, 323L, 49P

Popham, R., \& Narayan, R. 1995, ApJ, 442, 337

Proga, D. 2003a, ApJ, 585, 406

Proga, D. 2003b, ApJ, 592, L9

Ribiero, F.M.A. \& Diaz, M.P. 2007, AJ, 133, 2659

Rutten, R. G. M., van Paradijs, J., \& Tinbergen, J. 1992, A\&A, 260, 213 
Schwarzenberg-Czerny, A. \& Różyczka, M. 1988, Acta Astron., 38, 189

Shakura, N. I., \& Sunyaev, R. A. 1973, A\&A, 24, 337

Sion, E. M. 1985, ApJ, 297, 538

Sion, E. M., Long, K. S., Szkody, P., \& Huang, M. 1994, ApJ, 430, L53

Sion, E. M. 1995, ApJ, 438, 876

Sion, E. M. 1999, PASP, 111, 532

Smak, J. 2002, Acta Astron., 52, 263

Verbunt, F. 1987, A\&AS, 71, 339

Vrtilek, S. D., Silber, A., Raymond, J. C., \& Patterson, J. 1994, ApJ, 425, 787

Wade, R. A. 1988, ApJ, 335, 394

Warner, B. 1995, Cataclysmic Variable Stars (Cambridge: University Press)

Whitehurst, R. 1988, MNRAS, 233, 529

Whitehurst, R., \& King, A. 1991, MNRAS, 249, 25

Winter, L. \& Sion, E. M. 2003, ApJ, 582, 352

Woods, J. A., Verbunt, F., Cameron, A. Collier, Drew, J. E. \& Piters, A. 1992, MNRAS, 255,237 
Table 1: FUV Observations of V3885 Sgr: HST \& FUSE Spectra

\begin{tabular}{clcllll}
\hline $\begin{array}{c}\text { Date } \\
(\mathrm{dd} / \mathrm{mm} / \text { yyyy })\end{array}$ & $\begin{array}{l}\text { Telescope } \\
\text { /Instrument }\end{array}$ & $\begin{array}{c}\text { Exp time } \\
(\mathrm{sec})\end{array}$ & Dataset & $\begin{array}{l}\text { Filter/Grating } \\
\text { /Aperture }\end{array}$ & $\begin{array}{l}\text { Operation } \\
\text { Mode }\end{array}$ & $\begin{array}{l}\text { Calibration } \\
\text { Software }\end{array}$ \\
\hline 24 May 2000 & FUSE & 12392 & P1870101000 & LWRS & TTAG & CalFUSE 3.0 \\
30 Apr 2000 & STIS & 2195 & O5BI04010 & E140M/0.2x0.2 & TIME-TAG & CALSTIS 2.2 \\
\hline
\end{tabular}


Table 2. FUSE lines

\begin{tabular}{|c|c|c|c|}
\hline $\begin{array}{c}\text { Line } \\
\text { Identification }\end{array}$ & $\begin{array}{c}\text { Wavelength } \\
(\AA)\end{array}$ & $\begin{array}{c}\lambda_{0} \\
(\AA)\end{array}$ & Origin \\
\hline $\mathrm{H} \mathrm{I}$ & 914.13 & 914.29 & ism \\
\hline H I & 914.43 & 914.58 & ism \\
\hline $\mathrm{H} \mathrm{I}$ & 914.79 & 914.92 & ism \\
\hline H I & 915.19 & 915.33 & ism \\
\hline H I & 915.68 & 915.82 & ism \\
\hline H I & 916.30 & 916.43 & ism \\
\hline O I & 916.73 & 916.82 & ism \\
\hline H I & 917.05 & 917.18 & ism \\
\hline H I & 918.00 & 918.13 & ism \\
\hline H I & 919.22 & 919.35 & ism \\
\hline H I & 920.84 & 920.96 & ism \\
\hline H I & 921.75 & 921.86 & ism \\
\hline $\mathrm{H} \mathrm{I}$ & 923.01 & 923.15 & ism \\
\hline N IV & $\sim 922$ & 923.2 & $\mathrm{~s}$ \\
\hline O I & 924.83 & 924.95 & ism \\
\hline O I & 925.33 & 925.45 & ism \\
\hline H I & 926.11 & 926.23 & ism \\
\hline O I & 929.41 & 929.52 & ism \\
\hline O I & 930.13 & 930.26 & ism \\
\hline H I & 930.61 & 930.75 & ism \\
\hline S VI & $\sim 932$ & 933.38 & $\mathrm{~s}$ \\
\hline H I & 936.51 & 936.75 & ism \\
\hline H I & 937.67 & 937.80 & ism \\
\hline S VI & $\sim 943$ & 944.52 & $\mathrm{~S}$ \\
\hline $\mathrm{O}_{\mathrm{I}}$ & 948.57 & 948.69 & ism \\
\hline $\mathrm{H} \mathrm{I}$ & 949.61 & 949.74 & ism \\
\hline $\mathrm{O}_{\mathrm{I}}$ & 950.76 & 950.88 & ism \\
\hline $\mathrm{N}$ I+O I & 952.26 & & ism \\
\hline N I & 953.30 & 953.42 & ism \\
\hline
\end{tabular}


Table 2-Continued

\begin{tabular}{|c|c|c|c|}
\hline $\begin{array}{c}\text { Line } \\
\text { Identification }\end{array}$ & $\begin{array}{c}\text { Wavelength } \\
(\AA)\end{array}$ & $\begin{array}{l}\lambda_{0} \\
(\AA)\end{array}$ & Origin \\
\hline N I & 953.54 & 953.65 & ism \\
\hline N I & 953.87 & 953.97 & ism \\
\hline $\mathrm{N} \mathrm{I}$ & 953.97 & 954.10 & ism \\
\hline P II & 962.44 & 962.57 & ism \\
\hline $\mathrm{P}_{\text {II }}$ & 963.68 & 963.80 & ism \\
\hline N I & 963.87 & 963.99 & ism \\
\hline N I & 964.50 & 964.63 & ism \\
\hline N I & 964.94 & 965.04 & ism \\
\hline O I & 971.61 & 971.74 & ism \\
\hline $\mathrm{H} \mathrm{I}$ & 972.34 & 972.54 & ism \\
\hline C III & $\sim 976$ & 977.02 & $\mathrm{~S}$ \\
\hline C III & 976.88 & 977.02 & ism \\
\hline O I & 976.32 & 976.45 & ism \\
\hline O I & 988.56 & 988.65 & ism \\
\hline Si II & 989.75 & 989.87 & ism \\
\hline N III & $\sim 990$ & 991.5 & $\mathrm{~s}$ \\
\hline Si III & 997.26 & 997.38 & ism \\
\hline Si II & 1020.63 & 1020.70 & ism \\
\hline H I & 1025.37 & 1025.72 & $\mathrm{~s}, \mathrm{ism}$ \\
\hline H I & 1025.68 & 1025.72 & $\mathrm{~s}, \mathrm{ism}$ \\
\hline O VI & 1030.7 & 1031.93 & $\mathrm{~S}$ \\
\hline O VI & 1036.4 & 1037.62 & s \\
\hline C II & 1036.24 & 1036.34 & ism \\
\hline O I & 1039.18 & 1039.23 & ism \\
\hline Ar I & 1048.15 & 1048.22 & ism \\
\hline S IV & 1062.3 & 1062.65 & $\mathrm{~S}$ \\
\hline Ar I & 1066.59 & 1066.66 & ism \\
\hline S IV & 1072.5 & 1072.97 & $\mathrm{~S}$ \\
\hline N II & 1083.74 & 1083.99 & ism \\
\hline
\end{tabular}


Table 2 - Continued

\begin{tabular}{llll}
\hline \hline $\begin{array}{c}\text { Line } \\
\text { Identification }\end{array}$ & $\begin{array}{c}\text { Wavelength } \\
(\AA)\end{array}$ & \multicolumn{1}{c}{$\begin{array}{c}\lambda_{0} \\
(\AA)\end{array}$} & \multicolumn{1}{c}{ Origin } \\
\hline Si III & 1109.4 & 1108.36 & $\mathrm{~s}$ \\
& & 1109.94 & $\mathrm{~s}$ \\
Si III & 1113.0 & 1113.2 & $\mathrm{~s}$ \\
S IV & 1117.3 & 1117.76 & $\mathrm{~s}$ \\
P V & 1117.3 & 1117.98 & $\mathrm{~s}$ \\
& 1127.4 & 1128.01 & $\mathrm{~s}$ \\
Si IV & 1122.0 & 1122.5 & $\mathrm{~s}$ \\
& 1127.4 & 1128.3 & $\mathrm{~s}$ \\
N I & 1134.08 & 1134.16 & ism \\
N I & 1134.33 & 1134.42 & ism \\
N I & 1134.89 & 1134.98 & ism \\
C III & 1175.0 & $1174.9-1176.4$ & $\mathrm{~s}$ \\
\hline
\end{tabular}

Note. - $\mathrm{a}=$ possibly with terrestrial contamination; ism=ISM; $\mathrm{s}=$ source 
Table 3. STIS lines

\begin{tabular}{|c|c|c|c|}
\hline $\begin{array}{c}\text { Line } \\
\text { Identification }\end{array}$ & $\begin{array}{l}\text { Wavelength } \\
\qquad(\AA)\end{array}$ & $\begin{array}{l}\lambda_{0} \\
(\AA)\end{array}$ & Origin \\
\hline C III & 1173.62 & 1174.9-1176.4 & s \\
\hline \multirow[t]{2}{*}{ Si II } & 1190.36 & 1190.42 & ism \\
\hline & 1193.24 & 1193.29 & ism \\
\hline \multirow[t]{3}{*}{ N I } & 1199.49 & 1199.55 & ism \\
\hline & 1200.18 & 1200.22 & ism \\
\hline & 1200.67 & 1200.71 & ism \\
\hline Si III & 1204.77 & 1206.50 & $\mathrm{~s}$ \\
\hline Si III & 1206.45 & 1206.50 & ism \\
\hline H I & 1215.6 & 1215.67 & $\mathrm{~s}$ \\
\hline \multirow[t]{2}{*}{$\mathrm{N} \mathrm{V}$} & 1236.9 & 1238.82 & s \\
\hline & 1240.3 & 1242.80 & s \\
\hline \multirow[t]{2}{*}{$\mathrm{NV}$} & 1238.63 & 1238.82 & ism \\
\hline & 1242.59 & 1242.80 & ism \\
\hline \multirow[t]{3}{*}{ S II } & 1250.55 & 1250.58 & ism \\
\hline & 1253.77 & 1253.81 & ism \\
\hline & 1259.45 & 1259.52 & ism \\
\hline Si II & 1260.37 & 1260.42 & ism \\
\hline \multirow[t]{3}{*}{ Si III } & 1297.3 & 1294.55 & $\mathrm{~s}$ \\
\hline & & 1296.73 & $\mathrm{~s}$ \\
\hline & & 1298.89 & s \\
\hline O I & 1302.12 & 1302.17 & ism \\
\hline Si II & 1304.33 & 1304.37 & ism \\
\hline \multirow[t]{3}{*}{ C II } & 1334.47 & 1334.53 & ism \\
\hline & 1334.47 & 1334.66 & ism \\
\hline & 1335.66 & 1335.71 & ism \\
\hline \multirow[t]{2}{*}{ Si IV } & 1391.4 & 1393.76 & $\mathrm{~s}$ \\
\hline & 1400.35 & 1402.77 & $\mathrm{~S}$ \\
\hline Si II & 1526.62 & 1526.71 & ism \\
\hline C IV & $\sim 1545$ & 1548.20 & $\mathrm{~s}$ \\
\hline
\end{tabular}


Table 3-Continued

\begin{tabular}{llll}
\hline \hline $\begin{array}{c}\text { Line } \\
\text { Identification }\end{array}$ & $\begin{array}{c}\text { Wavelength } \\
(\AA)\end{array}$ & $\begin{array}{c}\lambda_{0} \\
(\AA)\end{array}$ & Origin \\
& & & \\
& & 1550.78 & $\mathrm{~s}$ \\
C IV & 1547.95 & 1548.20 & ism \\
& 1550.54 & 1550.78 & ism \\
He II & 1637.7 & 1640.5 & $\mathrm{~s}$ \\
Al II & 1670.73 & 1670.8 & ism \\
\hline
\end{tabular}

Note. - ism=ISM; s=source; there is possibly some atmospheric absorption contamination for N I, N II, Hi, O I. 
Table 4. V3885 Sgr Initial System Parameters

\begin{tabular}{llll}
\hline \hline parameter & \multicolumn{1}{c}{ value } & parameter & \multicolumn{1}{c}{ value } \\
\hline$M_{\mathrm{wd}}$ & $0.70 M_{\odot}{ }^{\mathrm{a}}$ & $i$ & $65^{\circ \mathrm{d}}$ \\
$M_{2}$ & $0.475 M_{\odot}^{\mathrm{b}}$ & $\dot{M}$ & $5.0 \times 10^{-9} M_{\odot} \mathrm{yr}^{-1} \mathrm{e}$ \\
$\mathrm{P}$ & 0.20716071 day $^{\mathrm{c}}$ & $d$ & $110 \pm 30 \mathrm{pc}^{\mathrm{f}}$ \\
\hline
\end{tabular}

anigge (2007) mean $M_{\mathrm{wd}}$; Hartley et al. (2005), $0.55 M_{\odot}$ to $0.8 M_{\odot} ;$ Ribiero \& Diaz (2007), $0.3 M_{\odot}$ to $0.9 M_{\odot}$

Knigge (2007) calibrated $P$ : $M_{2}$ relation; Hartley et al. (2005), $q=0.6$ to 0.8 ; Ribiero \& Diaz (2007), $M_{2}=0.2 M_{\odot}$ to $0.55 M_{\odot}$

Ribiero \& Diaz (2007)

Haug \& Dreschel (1985), $i$ between $60^{\circ}$ and $70^{\circ}$; Ribiero \& Diaz (2007), $i$ between $45^{\circ}$ and $75^{\circ}$; Hartley et al. $(2005), i>65^{\circ}$

Einnell et al. (2007) and similarity to IX Vel (Hartley et al. (2002))

${ }^{\mathrm{f}}$ Hipparcos value 
Table 5. Properties of accretion disk with mass transfer rate $\dot{M}=5.0 \times 10^{-9} M_{\odot} \mathrm{yr}^{-1}$ and WD mass of $0.70 M_{\odot}$.

\begin{tabular}{rrccccc}
\hline \hline$r / r_{\text {wd }, 0}$ & \multicolumn{1}{c}{$T_{\text {eff }}$} & $m_{0}$ & $\log g$ & $z_{0}$ & $N e$ & $\tau_{\text {Ross }}$ \\
\hline 1.36 & 53136 & $1.221 \mathrm{E} 4$ & 6.84 & $8.27 \mathrm{E} 7$ & $2.0 \mathrm{E} 17$ & $2.33 \mathrm{E} 4$ \\
2.00 & 47642 & $1.491 \mathrm{E} 4$ & 6.58 & $1.43 \mathrm{E} 8$ & $1.3 \mathrm{E} 17$ & $2.78 \mathrm{E} 4$ \\
3.00 & 38525 & $1.456 \mathrm{E} 4$ & 6.28 & $2.43 \mathrm{E} 8$ & $7.7 \mathrm{E} 16$ & $3.35 \mathrm{E} 4$ \\
4.00 & 32380 & $1.356 \mathrm{E} 4$ & 6.06 & $3.49 \mathrm{E} 8$ & $5.1 \mathrm{E} 16$ & $3.92 \mathrm{E} 4$ \\
5.00 & 28086 & $1.259 \mathrm{E} 4$ & 5.89 & $4.60 \mathrm{E} 8$ & $3.8 \mathrm{E} 16$ & $4.52 \mathrm{E} 4$ \\
6.00 & 24918 & $1.176 \mathrm{E} 4$ & 5.75 & $5.76 \mathrm{E} 8$ & $3.0 \mathrm{E} 16$ & $5.16 \mathrm{E} 4$ \\
8.00 & 20531 & $1.043 \mathrm{E} 4$ & 5.53 & $8.14 \mathrm{E} 8$ & $2.5 \mathrm{E} 16$ & $6.56 \mathrm{E} 4$ \\
10.00 & 17612 & $9.439 \mathrm{E} 3$ & 5.35 & $1.06 \mathrm{E} 9$ & $1.9 \mathrm{E} 16$ & $8.05 \mathrm{E} 4$ \\
12.00 & 15512 & $8.664 \mathrm{E} 3$ & 5.21 & $1.32 \mathrm{E} 9$ & $1.5 \mathrm{E} 16$ & $9.53 \mathrm{E} 4$ \\
14.00 & 13923 & $8.040 \mathrm{E} 3$ & 5.09 & $1.58 \mathrm{E} 9$ & $1.2 \mathrm{E} 16$ & $1.08 \mathrm{E} 5$ \\
16.00 & 12670 & $7.526 \mathrm{E} 3$ & 4.98 & $1.85 \mathrm{E} 9$ & $9.9 \mathrm{E} 15$ & $1.18 \mathrm{E} 5$ \\
18.00 & 11653 & $7.092 \mathrm{E} 3$ & 4.89 & $2.12 \mathrm{E} 9$ & $8.3 \mathrm{E} 15$ & $1.23 \mathrm{E} 5$ \\
20.00 & 10810 & $6.721 \mathrm{E} 3$ & 4.80 & $2.38 \mathrm{E} 9$ & $6.7 \mathrm{E} 15$ & $1.23 \mathrm{E} 5$ \\
24.00 & 9487 & $6.115 \mathrm{E} 3$ & 4.65 & $2.92 \mathrm{E} 9$ & $5.1 \mathrm{E} 15$ & $1.17 \mathrm{E} 5$ \\
28.00 & 8491 & $5.638 \mathrm{E} 3$ & 4.51 & $3.57 \mathrm{E} 9$ & $4.7 \mathrm{E} 15$ & $1.07 \mathrm{E} 5$ \\
32.00 & 7711 & $5.251 \mathrm{E} 3$ & 4.46 & $4.90 \mathrm{E} 9$ & $2.2 \mathrm{E} 14$ & $9.32 \mathrm{E} 4$ \\
\hline
\end{tabular}

Note. - Each line in the table represents a separate annulus. A Shakura \& Sunyaev (1973) viscosity parameter $\alpha=0.1$ was used in calculating all annuli. The WD radius, $r_{\mathrm{wd}, 0}$, is the radius, $0.01091 R_{\odot}$, of a zero temperature Hamada-Salpeter carbon model. See the text (§4.) for a discussion of the table units. 
Table 6. Temperature profile for V3885 Sgr accretion disk with mass transfer rate of $\dot{M}=5.0 \times 10^{-9} M_{\odot} \mathrm{yr}^{-1}$ and WD mass of $0.70 M_{\odot}$.

\begin{tabular}{rcrrrr}
\hline \hline$r / r_{\text {wd }}$ & $T_{\text {eff }}$ & $r / r_{\mathrm{wd}}$ & $T_{\text {eff }}$ & $r / r_{\mathrm{wd}}$ & $T_{\text {eff }}$ \\
\hline 1.00 & 43608 & 13.32 & 12308 & 27.94 & 7260 \\
1.18 & 43608 & 14.65 & 11511 & 29.27 & 7021 \\
1.36 & 45358 & 15.98 & 10826 & 30.59 & 6799 \\
2.69 & 34985 & 17.31 & 10229 & 31.92 & 6594 \\
4.02 & 27556 & 18.64 & 9705 & 33.25 & 6402 \\
5.35 & 22948 & 19.96 & 9240 & 34.58 & 6223 \\
6.68 & 19807 & 21.29 & 8824 & 35.91 & 6055 \\
8.01 & 17518 & 22.62 & 8449 & 37.24 & 5898 \\
9.33 & 15767 & 23.95 & 8110 & 38.57 & 5749 \\
10.66 & 14380 & 25.28 & 7802 & 39.90 & 5610 \\
11.99 & 13249 & 26.61 & 7520 & 41.22 & 5478 \\
\hline
\end{tabular}

Note. - The WD radius $r_{\mathrm{wd}}$ is the radius of a $60,000 \mathrm{~K} 0.70 M_{\odot}$ WD interpolated from Table 4a of Panei, Althaus, \& Benvenuto (2000). The $T_{\text {eff }}=43608 \mathrm{~K}$ for $r / r_{\mathrm{wd}}=1.00$ refers to the inner edge of the innermost annulus. The same $T_{\text {eff }}$ for $r / r_{\mathrm{wd}}=1.18$ refers to the outer edge of the innermost annulus which coincides with the inner edge of the next annulus. The remaining $T_{\text {eff }}$ values refer to the inner edge of the corresponding annuli. 
Table 7. Values of $\widetilde{\chi}^{2}$ as function of model parameters

\begin{tabular}{cccccccc}
\hline \hline$\dot{M}\left(\mathrm{x} 10^{-9} M_{\odot} / \mathrm{yr}\right)$ & $T_{\text {wd }}(\mathrm{K})$ & $i(\mathrm{deg})$ & $\tilde{\chi}^{2}$ & $\dot{M}\left(\mathrm{x} 10^{-9} M_{\odot} / \mathrm{yr}\right)$ & $T_{\mathrm{wd}}(\mathrm{K})$ & $i(\mathrm{deg})$. & $\tilde{\chi}^{2}$ \\
\hline 4.0 & 70,000 & 65 & 7.51 & 5.0 & 57,000 & 64 & 3.95 \\
4.0 & 75,000 & 65 & 5.60 & 5.0 & 57,000 & 66 & 4.43 \\
4.5 & 57,000 & 65 & 6.86 & 5.0 & 57,000 & 70 & 39.73 \\
4.5 & 60,000 & 65 & 5.05 & 5.2 & 40,000 & 65 & 4.69 \\
4.5 & 65,000 & 65 & 3.93 & 5.2 & 45,000 & 65 & 3.14 \\
4.5 & 70,000 & 65 & 3.14 & 5.2 & 50,000 & 65 & 2.91 \\
4.5 & 75,000 & 65 & 3.97 & 5.2 & 52,000 & 65 & 2.73 \\
4.8 & 52,000 & 65 & 5.22 & 5.2 & 57,000 & 65 & 3.04 \\
4.8 & 57,000 & 65 & 3.42 & 5.2 & 60,000 & 65 & 4.41 \\
4.8 & 60,000 & 65 & 3.00 & 5.5 & 35,000 & 65 & 4.34 \\
4.8 & 65,000 & 65 & 3.40 & 5.5 & 40,000 & 65 & 3.08 \\
4.8 & 70,000 & 65 & 4.26 & 5.5 & 45,000 & 65 & 3.19 \\
5.0 & 45,000 & 65 & 4.54 & 5.5 & 50,000 & 65 & 3.24 \\
5.0 & 50,000 & 65 & 4.25 & 5.5 & 52,000 & 65 & 3.93 \\
5.0 & 52,000 & 65 & 3.39 & 5.5 & 57,000 & 65 & 5.93 \\
5.0 & 57,000 & 65 & 2.65 & 6.0 & 35,000 & 65 & 4.03 \\
5.0 & 60,000 & 65 & 3.44 & 6.0 & 40,000 & 65 & 6.48 \\
5.0 & 65,000 & 65 & 5.80 & 6.0 & 45,000 & 65 & 4.13 \\
5.0 & 57,000 & 60 & 49.94 & 6.0 & 50,000 & 65 & 9.88 \\
\hline
\end{tabular}


Table 8. V3885 Sgr Model System Parameters

\begin{tabular}{llll}
\hline \hline parameter & \multicolumn{1}{c}{ value } & parameter & \multicolumn{1}{c}{ value } \\
\hline$M_{\mathrm{wd}}$ & $0.70 \pm 0.1 M_{\odot}$ & $T_{\mathrm{eff}, \mathrm{s}}($ pole $)$ & $3650 \pm 100 \mathrm{~K}$ \\
$M_{\mathrm{s}}$ & $0.475 M_{\odot}$ & $T_{\mathrm{eff}, \mathrm{s}}($ point $)$ & $2214 \mathrm{~K}$ \\
$\dot{M}$ & $5.0 \pm 2.0 \times 10^{-9} M_{\odot} \mathrm{yr}^{-1}$ & $T_{\mathrm{eff}, \mathrm{s}}($ side $)$ & $3598 \mathrm{~K}$ \\
$\mathrm{P}$ & 0.2071607 days & $T_{\mathrm{eff}, \mathrm{s}}(\mathrm{back})$ & $3489 \mathrm{~K}$ \\
$D$ & $1.55396 R_{\odot}$ & $r_{s}($ pole $)$ & $0.209 R_{\odot}$ \\
$\Omega_{\mathrm{wd}}$ & 116.0 & $r_{s}($ point $)$ & $0.296 R_{\odot}$ \\
$\Omega_{s}$ & 3.2053112 & $r_{s}($ side $)$ & $0.218 R_{\odot}$ \\
$i$ & $65 \pm 2^{\circ}$ & $r_{s}$ back $)$ & $0.239 R_{\odot}$ \\
$T_{\mathrm{eff}, \mathrm{wd}}$ & $57,000 \pm 5000 \mathrm{~K}$ & $\log g_{s}($ pole $)$ & 4.73 \\
$r_{\mathrm{wd}}$ & $0.0134 R_{\odot}$ & $\log g_{s}($ point $)$ & -.390 \\
$\log g_{\mathrm{wd}}$ & 8.02 & $\log g_{s}($ side $)$ & 4.65 \\
$A_{\mathrm{wd}}$ & 1.0 & $\log g_{s}(\mathrm{back})$ & 4.48 \\
$A_{s}$ & 0.6 & $r_{a}$ & $0.56 R_{\odot}$ \\
$\beta_{\mathrm{wd}}$ & 0.25 & $r_{b}$ & $0.01091 R_{\odot}$ \\
$\beta_{s}$ & 0.08 & $H$ & $0.10 R_{\odot}$ \\
\hline
\end{tabular}

Note. - wd refers to the WD; $s$ refers to the secondary star. $D$ is the component separation of centers, $\Omega$ is a Roche potential. Temperatures are polar values, $A$ values are bolometric albedos, and $\beta$ values are gravity-darkening exponents. $r_{a}$ specifies the outer radius of the accretion disk, set at the tidal cut-off radius, and $r_{b}$ is the accretion disk inner radius, as determined in the final system model, while $H$ is the semi-height of the accretion disk rim. 
Table 9. Parameters of ISM model

\begin{tabular}{ll}
\hline \hline Parameter & \multicolumn{1}{c}{ Value } \\
\hline $\mathrm{N}(\mathrm{H})$ & $1.0 \times 10^{18} \mathrm{~cm}^{-2}$ \\
$\mathrm{D} / \mathrm{H}$ & $2 \times 10^{-5}$ \\
$\mathrm{~N}(\mathrm{H} 2)$ & $1 \times 10^{14} \mathrm{~cm}^{-2}$ \\
vel. & $-50.0 \mathrm{~km} \mathrm{~s}^{-1}$ \\
temp. & $250 \mathrm{~K}$ \\
metallicity & $1.0(1.0=$ solar $)$ \\
\hline
\end{tabular}



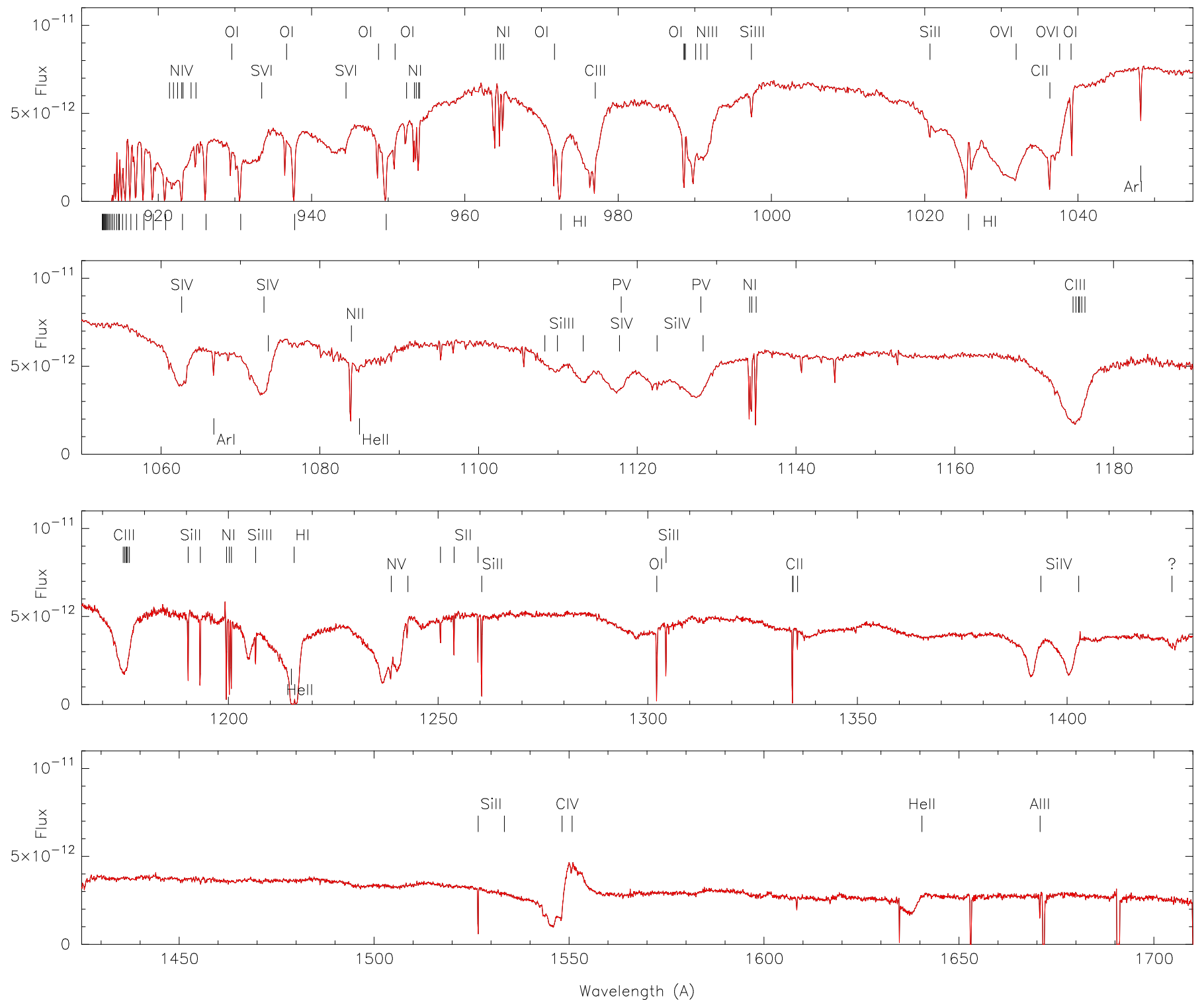

Fig. 1.- FUSE plus STIS combined spectra with line identifications. 

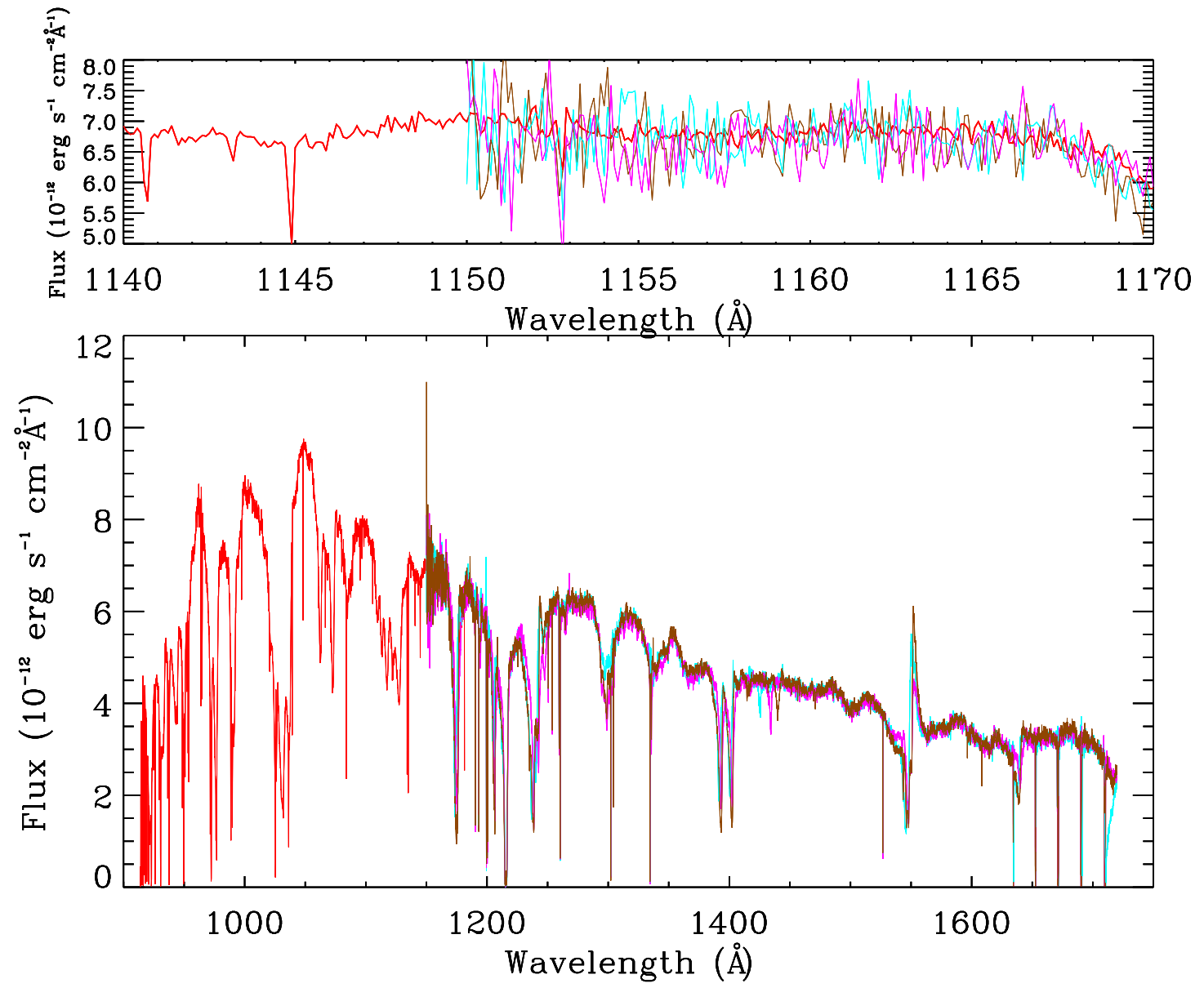

Fig. 2.- Combined FUSE and STIS spectra. The upper panel shows the fit of the spectra in the overlap region when small normalizing factors are applied to the STIS spectra. The FUSE spectrum is red; the first STIS spectrum (Table 1), divided by 0.98 , is cyan; the second STIS spectrum, divided by 1.12, is magenta; the third STIS spectrum, divided by 0.94, is brown. Note the accurate superposition of the STIS spectra with application of normalizing factors. 


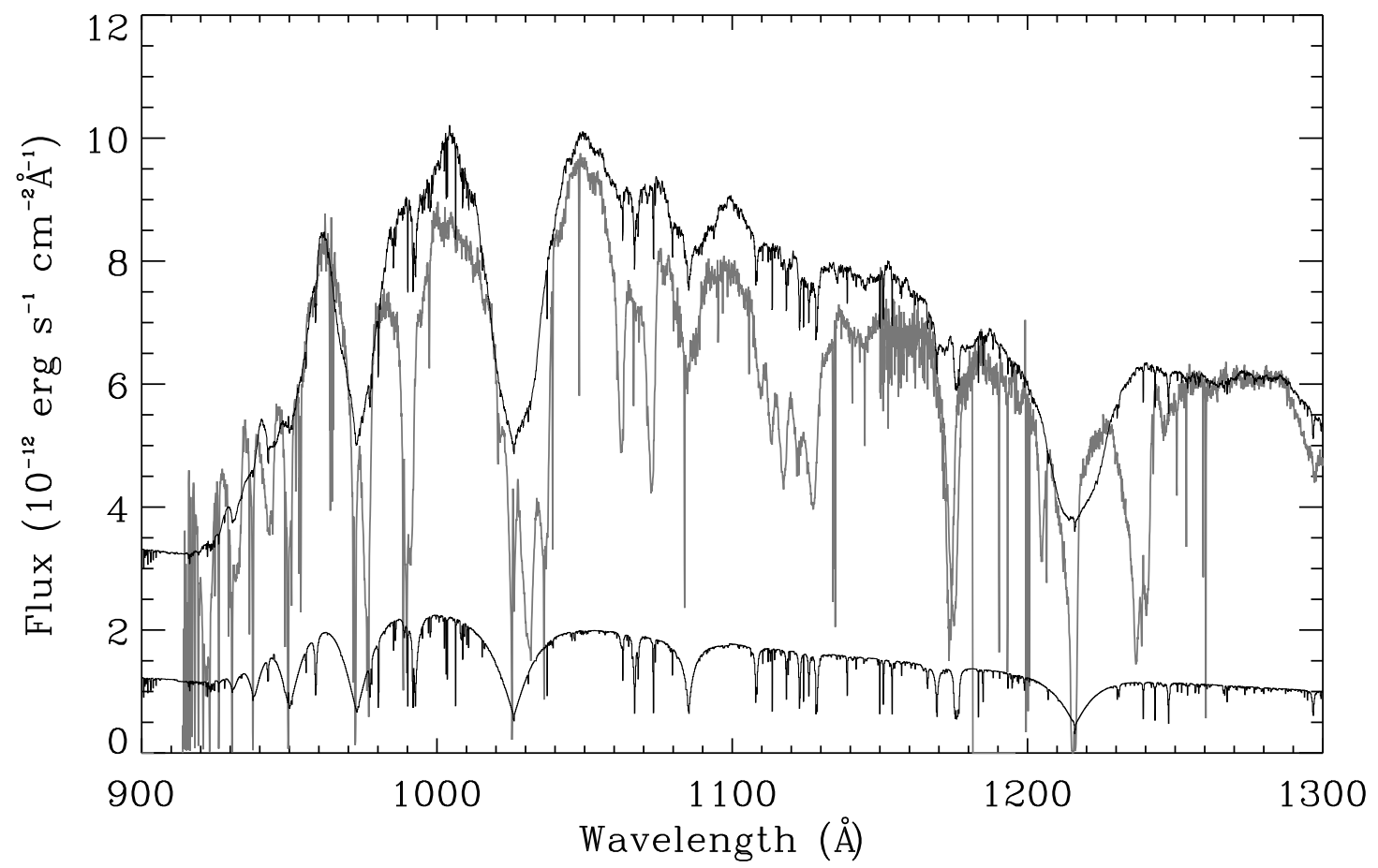

Fig. 3.- FUV fit of system synthetic spectrum to FUSE and STIS combined spectrum (gray plot) of V3885Sgr. Note the extensive absorption by local highly ionized material. The lower spectrum is the contribution of a $57,000 \mathrm{~K}$ WD; the top synthetic spectrum is the system synthetic spectrum including the WD and a $\dot{M}=5.0 \times 10^{-9} M_{\odot} \mathrm{yr}^{-1}$ standard model accretion disk. See the text for a discussion. 


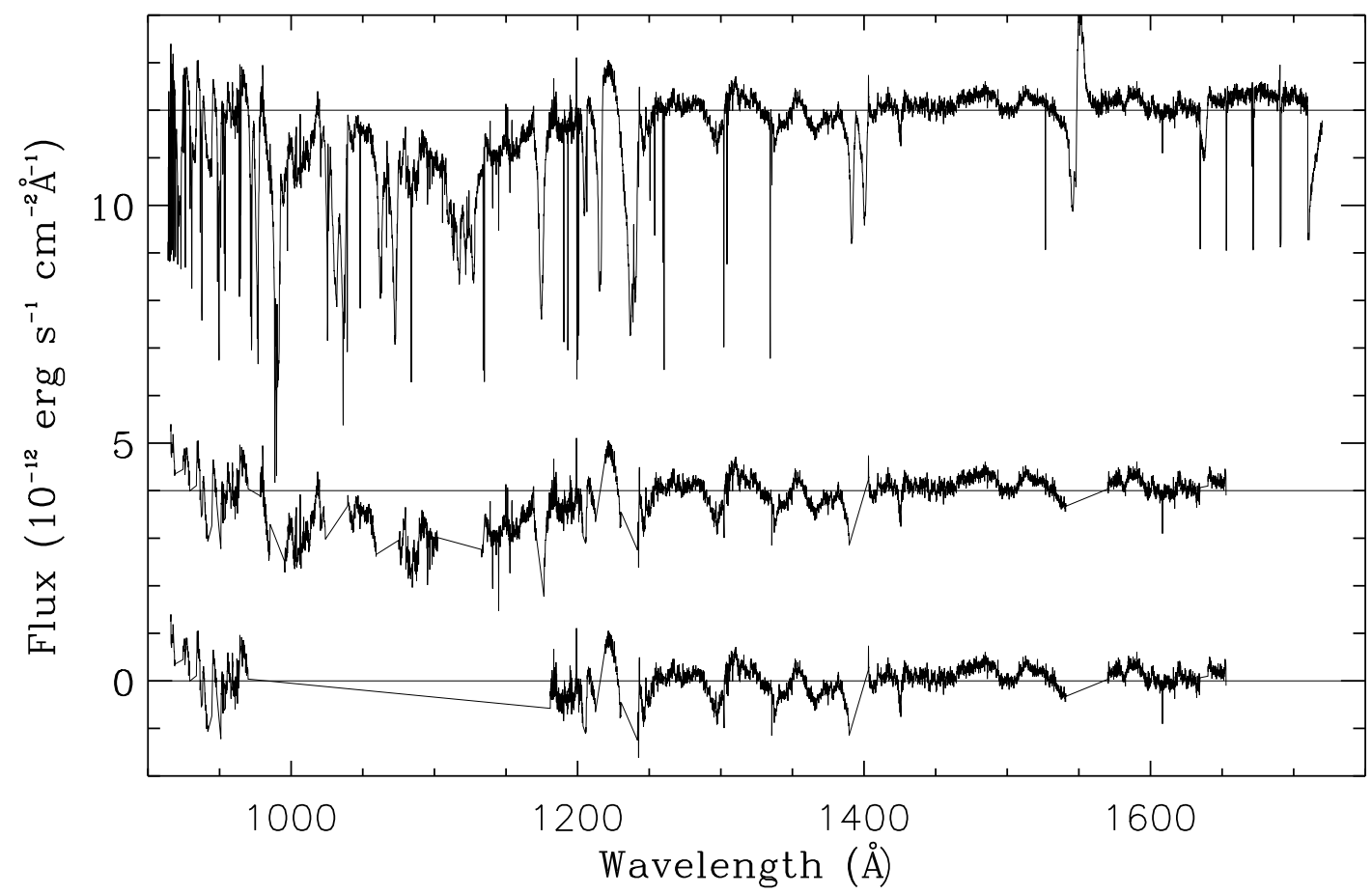

Fig. 4.- Plots of residuals from the visually selected best model. The top plot, displaced upward by 12.0 ordinate units, is the residuals in the unmasked case. The middle plot, displaced upward by 4.0 ordinate units, is the residuals for a partially masked observed spectrum. The bottom plot is the residuals for the finally adopted masked case. Note the three horizontal lines marking residual values of 0.0 for the three cases. See the text for a discussion. 


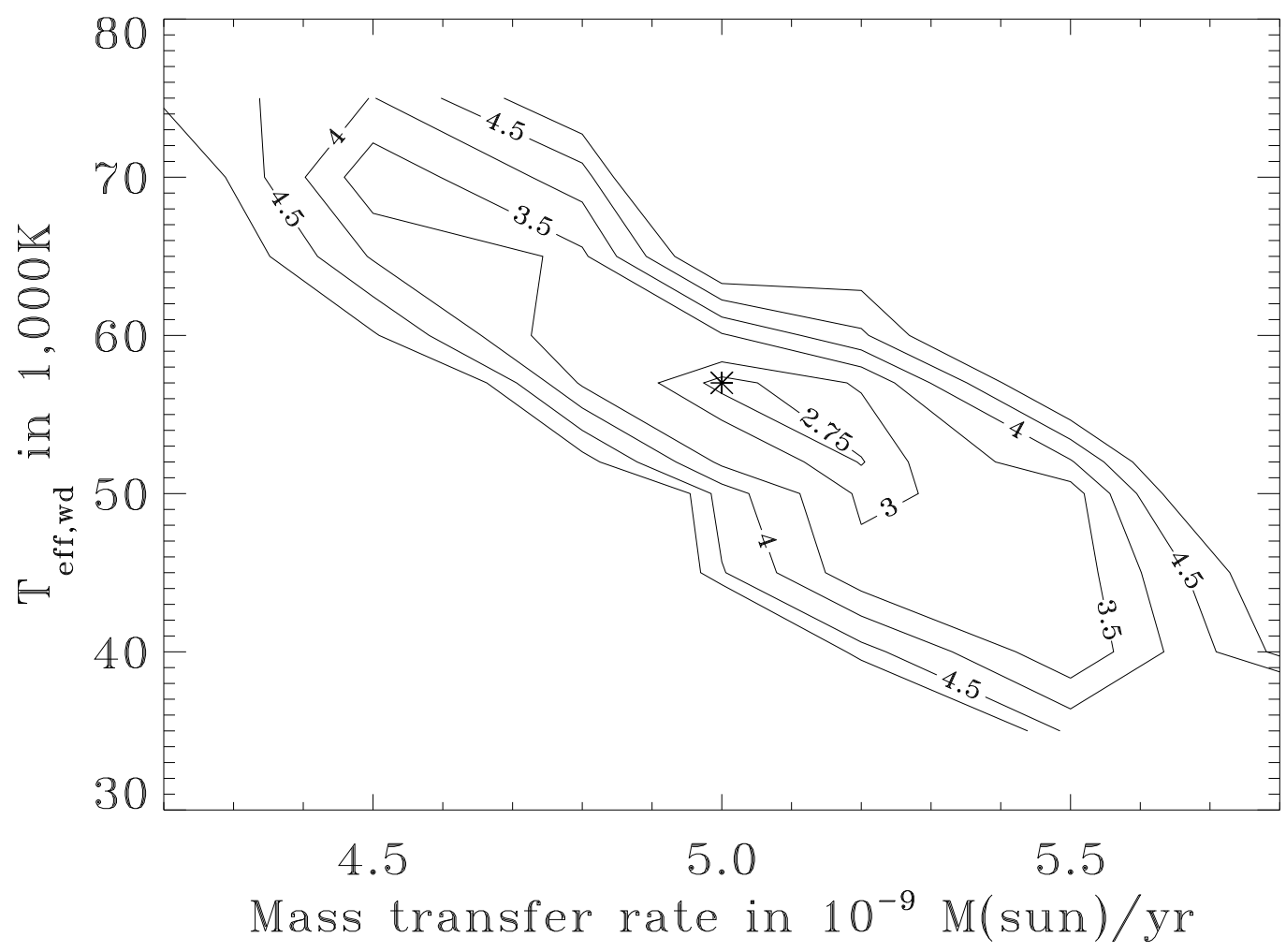

Fig. 5.- Contour plot showing values of $\widetilde{\chi}^{2}$ in the $\dot{M}, T_{\text {eff,wd }}$ plane for the system models tabulated in Table 7 . An asterisk marks the $\widetilde{\chi}^{2}$ value for the preferred solution. See the text for a discussion. 


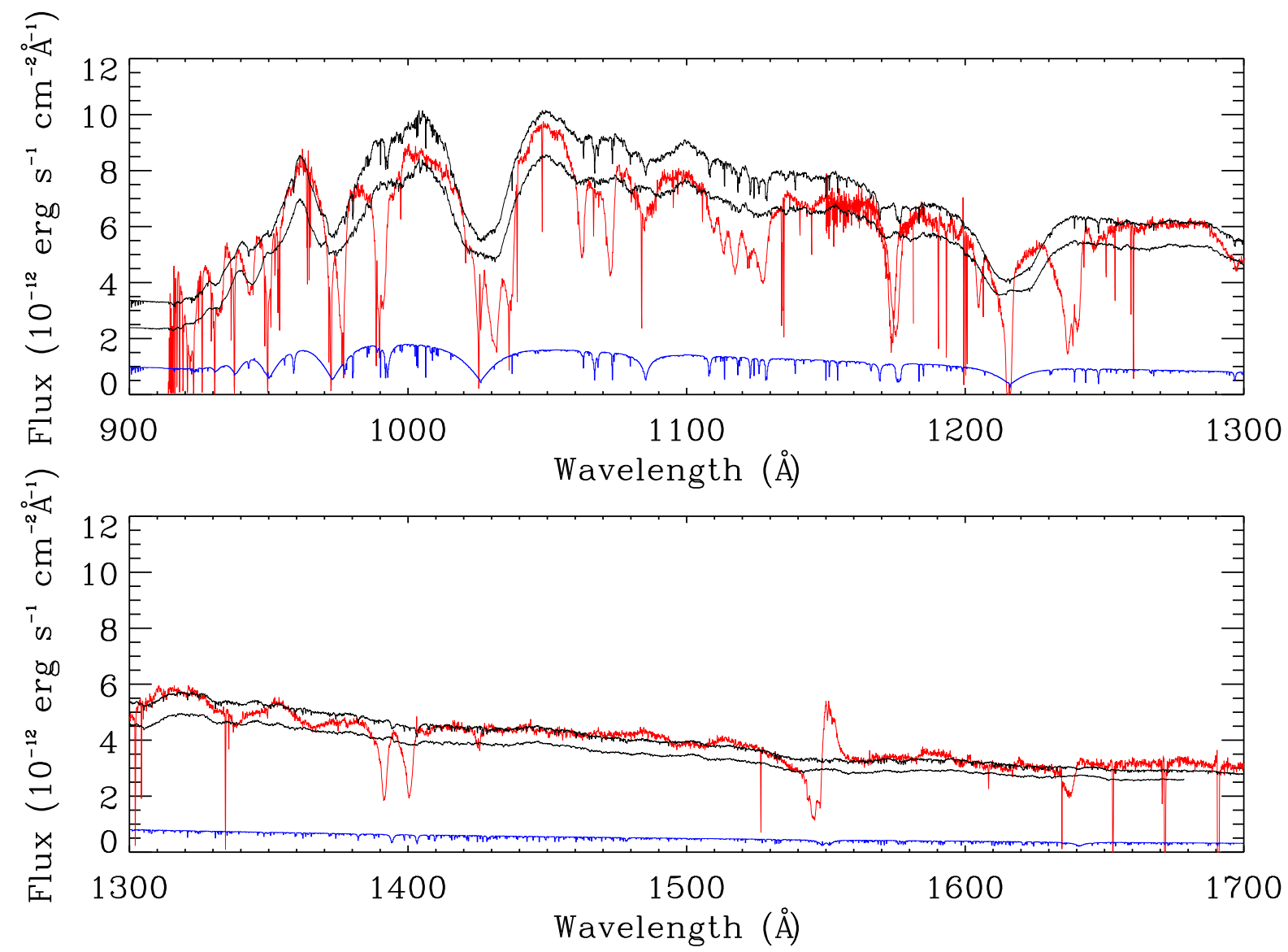

Fig. 6.- As in Figure 3 but for the full wavelength range of the combined spectra. The synthetic spectrum of the accretion disk alone is the next-to-top synthetic spectrum. The uppermost system synthetic spectrum is the sum of the accretion disk and the WD (blue line). The contributions of the secondary star and accretion disk rim are negligible. 


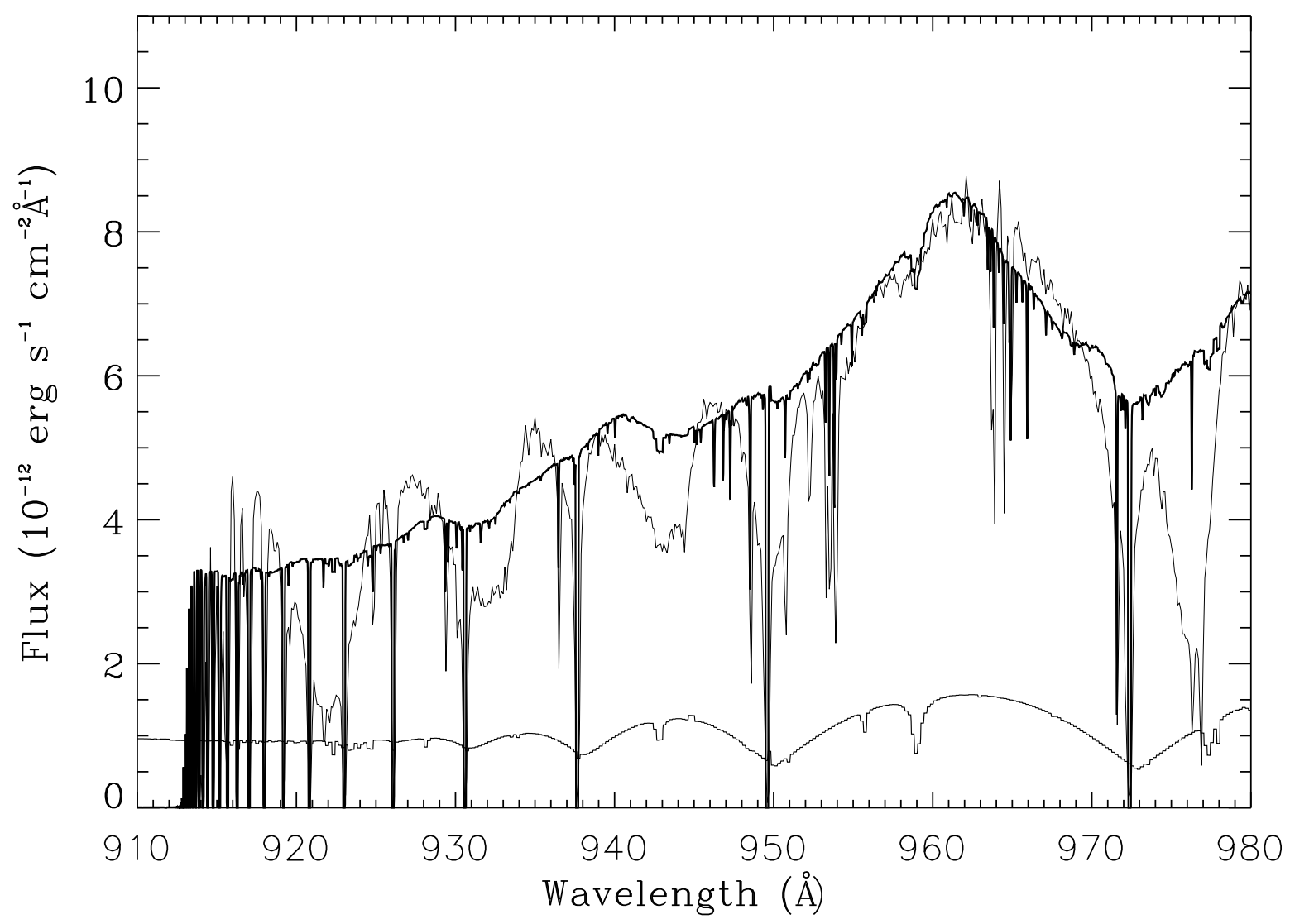

Fig. 7.- The model of Figure 6 but with ISM correction. Note the cutoff at $912 \AA$. 